\title{
CONFIGURATIONS AND INVARIANT NETS FOR AMENABLE HYPERGROUPS AND RELATED ALGEBRAS
}

\author{
BENJAMIN WILLSON
}

Abstract. Let $H$ be a hypergroup with left Haar measure. The amenability of $H$ can be characterized by the existence of nets of positive, norm one functions in $L^{1}(H)$ which tend to left invariance in any of several ways. In this paper we present a characterization of the amenability of $H$ using configuration equations. Extending work of Rosenblatt and Willis, we construct, for a certain class of hypergroups, nets in $L^{1}(H)$ which tend to left invariance weakly, but not in norm.

We define the semidirect product of $H$ with a locally compact group. We show that the semidirect product of an amenable hypergroup and an amenable locally compact group is an amenable hypergroup and show how to construct Reiter nets for this semidirect product.

These results are generalized to Lau algebras, providing a new characterization of left amenability of a Lau algebra and a notion of a semidirect product of a Lau algebra with a locally compact group. The semidirect product of a left amenable Lau algebra with an amenable locally compact group is shown to be a left amenable Lau algebra.

\section{Preliminaries}

1.1. Introduction. A hypergroup is a locally compact space with a convolution product mapping each pair of points to a probability measure with compact support. Hypergroups are a generalization of locally compact groups wherein the convolution of two points corresponds to the point evaluation measure at their product. The abstract study of hypergroups began in the 1970s with Dunkl [7, Jewett [18, and Spector [42. A detailed treatment can be found in the text of Bloom and Heyer [4. Numerous authors continue to study various aspects of hypergroups including amenability properties [22,24, 25, 41], Fourier transforms and spaces [33, 45], other function spaces [10, 11, 21, 47, and other aspects [8, 13, 37, 39, 44. Within the literature, there is some variation in the precise definition of a hypergroup, but this paper will use the definition of Jewett.

In this paper we examine nets of functions on hypergroups tending to left invariance. Such nets approximate left invariant means and have previously been studied for amenable groups, semigroups, and certain Banach algebras [14, 27, 28, 30. Similar nets are relevant to character amenability and other conditions related to amenability of hypergroups and Lau algebras (see $[2,3,9,12,15,19,23,32$ ).

Received by the editors June 1, 2011 and, in revised form, October 22, 2011.

2010 Mathematics Subject Classification. Primary 43A62; Secondary 43A07, 20N20.

Key words and phrases. Semidirect product, hypergroup, amenable, configurations, Lau algebra, invariant net, Reiter net.

The financial assistance of NSERC in the form of a Postgraduate Scholarship(Doctoral) and funding through a Discovery Grant awarded to Professor Anthony To-Ming Lau is gratefully acknowledged. 
The existence of a left Haar measure for every hypergroup remains an open question, however, it is known that such a measure exists if $H$ is commutative [2], compact [18, or discrete [18. Skantharajah [41] showed that if $H$ is a hypergroup which admits a left Haar measure, then the function spaces of $U C B_{r}(H), U C B(H)$, $C(H)$, and $L^{\infty}(H)$ all either admit a left invariant mean (if $H$ is amenable) or all do not. Using the approach of Namioka [34] it is apparent that amenability can be characterized by the existence of a net of positive norm one elements of $L^{1}(H)$ which tend to left invariance with respect to either the weak or norm topologies. Skantharajah and Lasser have shown that amenability of $H$ can also be characterized by any of several Reiter conditions [25,41]. As well, Singh [40] has published some results relating to Reiter conditions for hypergroups.

A net of positive norm one functions in $L^{1}(H)$ which tends to left invariance in norm must also tend to left invariance in the weak topology, but the converse is not generally true. In section 2, we investigate the question of when nets exist which tend to left invariance weakly, but not in norm, and how such nets can be constructed. In 36, Rosenblatt and Willis constructed such nets for infinite locally compact groups. In so doing, they introduced the notion of configurations and related systems of linear equations associated to a measurable partition and finite subset of the group. Motivated by their approach, we introduce configurations for hypergroups. We show in Theorem 2.6 that $H$ is amenable if and only if for every choice of partition and subset, the configuration equations have a positive, normalized, inequality preserving solution. This generalizes proposition 3.2 of [36] from locally compact groups to all hypergroups. Due to the properties of translation being fundamentally different between groups and general hypergroups, the method of proof for the generalization is significantly different from that given by Rosenblatt and Willis, and so the generalized result does not lend itself to constructing the nets of interest. It is interesting in other respects, however, since it provides a new characterization of amenability of hypergroups and indeed can be extended to characterize the existence of left invariant means on other function spaces. We conclude this section by using the result of Rosenblatt and Willis to construct, for a large class of double coset spaces of locally compact amenable groups, nets of positive, norm one $L^{1}$ functions which tend to left invariance in a weak sense, but not in norm.

In section 3 of the paper, we introduce the semidirect product of a hypergroup with a locally compact group of automorphisms of the hypergroup. In particular, we show that the semidirect product of an amenable hypergroup with an amenable locally compact group is itself an amenable hypergroup. This result is shown using a similar method to that used in [46] and demonstrates that Reiter nets for a locally compact group and a hypergroup can be combined to form a Reiter net for the semidirect product.

In section 4, we consider the more general class of Lau algebras (called $\mathrm{F}$ algebras in [26]) which contain the measure algebras of groups, hypergroups and semigroups. Left amenability of a Lau algebra has some known characterizations involving the existence of nets which tend to left invariance [26, 29. We show that the constructions we have presented thus far - those of configurations and of semidirect products - have analogous concepts for Lau algebras. Using slight modifications of the proofs of earlier sections, we provide a new characterization of left amenability of a Lau algebra and show that the semidirect product of a Lau algebra and a 
locally compact group is again a Lau algebra. We further show that if the Lau algebra is left amenable and the group is amenable, then the semidirect product is left amenable.

We conclude the paper with some remarks on possible generalizations of these results.

1.2. Definitions. In this paper we use the notation of Jewett [18] (although he referred to hypergroups as convos) with the following modifications:

- We denote a point mass measure at $x$ by $\delta_{x}$.

- We denote the positive elements of norm one of a space $A$ by $A_{1}^{+}$.

- We denote the involution of $x$ by $\check{x}$.

- We denote the characteristic function of a set $S$ by $\chi_{S}$.

Definition 1.1 (See [18 or 4] for more details). A non-empty locally compact Hausdorff space, $H$, is called a hypergroup if

- $M(H)$ is a complex algebra with + and $*$;

- there exists a unique $e \in H$ such that for every $x \in H, \delta_{e} * \delta_{x}=\delta_{x} * \delta_{e}=\delta_{x}$;

- there exists an involution $x \mapsto \check{x}$ which is a homeomorphism of $H$ onto itself with $\check{\check{x}}=x \forall x \in H, e \in \operatorname{supp} \delta_{x} * \delta_{y}$ if and only if $y=\check{x}$, and $(\mu * \nu)^{\check{\nu}}=\check{\nu} * \check{\mu}$, where $\check{\mu}(A)=\mu(\check{A})$ for any Borel subset $A$;

- the bilinear mapping

$$
\begin{aligned}
*: M(H) \times M(H) & \rightarrow M(H), \\
(\mu, \nu) & \mapsto \mu * \nu
\end{aligned}
$$

is non-negative and $*_{M(H)+\times M(H)}$ is weak-weak continuous;

- for $x, y \in H$ the product of the point evaluation measures $\delta_{x} * \delta_{y}$ is in $M(H)_{1}^{+}$and has compact support; and

- the mapping $(x, y) \mapsto \operatorname{supp} \delta_{x} * \delta_{y}$ is continuous from the product topology on $H \times H$ to the Michael topology on the compact subset of $H$.

Let $f$ be a Borel function on $H$ and $\mu \in M(H)$. We define the left translation $\mu * f$ by $\mu * f(x)=\check{\mu} * \delta_{x}(f)$.

We say that $H$ is amenable if there exists a positive linear functional of norm 1 on $C(H)$ which is invariant under left translation.

Definition 1.2. A left Haar measure for $H$ is a non-zero regular Borel measure (with values in $[0, \infty]) \lambda$ which is left-invariant in the sense that for any $f \in C_{C}(H)$, we have that $\lambda\left(\delta_{x} * f\right)=\lambda(f)$ for all $x \in H$.

Remark 1.1. It remains an open question whether every hypergroup admits a left Haar measure. In particular, it remains unknown whether every amenable hypergroup admits a left Haar measure.

Remark 1.2. If $H$ does admit a left Haar measure $\lambda$, however, it is unique up to a scalar multiple [18. Because of this, for hypergroups with left Haar measures we define the standard $L^{p}(H, \lambda)$ function spaces, often omitting the $\lambda$.

Definition 1.3. We say that a continuous function $f \in C(H)$ is right uniformly continuous if the map

$$
H \ni x \mapsto \delta_{x} * f
$$

is continuous in norm. We denote the collection of right uniformly continuous functions on $H$ by $U C B_{r}(H)$. 
Remark 1.3. Skantharajah [41] showed that for hypergroups with left Haar measure, $U C B_{r}(H)=L^{1}(H) * L^{\infty}(H)$.

Remark 1.4. A hypergroup with left Haar measure is amenable if and only if it admits a net of positive, norm one functions $\left(f_{\alpha}\right)_{\alpha} \in L^{1}(H)$ which are asymptotically left invariant in one of the following ways:

(1) For all $x \in H, \delta_{x} * f_{\alpha}-f_{\alpha} \rightarrow 0$ in the weak topology of $L^{1}(H)$.

(2) For all $x \in H, \delta_{x} * f_{\alpha}-f_{\alpha} \rightarrow 0$ in the norm topology of $L^{1}(H)$.

(3) The net is a Reiter net. That is, for any compact $K \subset H$ and $\varepsilon>0$ there exists $\alpha_{0}$ such that for $\alpha \geq \alpha_{0}$,

$$
\left\|\delta_{x} * f_{\alpha}-f_{\alpha}\right\|<\varepsilon \quad \forall x \in K .
$$

\section{INVARIANT NETS FROM CONFIGURATION EQUATIONS}

In 36, Rosenblatt and Willis introduced the notion of a configuration and the configuration equations corresponding to a locally compact group to investigate certain properties of groups, particularly a characterization of amenability. Using this characterization, they constructed a net satisfying condition (1), but not (2), from Remark 1.4 for any infinite, amenable, locally compact group. Configurations have also been used to study other group properties in 1 .

In the group setting, we begin with a finite partition, or colouring of $G$, a locally compact group into $m$ measurable subsets, $\left\{E_{1}, \ldots, E_{m}\right\}$, and a selection of $n$ group elements, $\left\{g_{1}, \ldots, g_{n}\right\}$. A configuration $C=\left(C_{0}, C_{1}, \ldots, C_{n}\right)$ is an ordered choice of $n+1$ (not necessarily distinct) colours $\left(E_{i} \mathrm{~s}\right)$. $C$ is realized by $\left(x_{0}, x_{1}, \ldots, x_{n}\right) \in$ $G^{n+1}$ if $x_{j} \in C_{j}$ for $j=0, \ldots, n$ and $x_{j}=g_{j} x_{0}$ for $j=1, \ldots, n$.

In [36] the notation $x_{j}(C)$ is used to denote the points which occur in the $j$ th element of a realization of $C$.

This approach cannot be immediately extended to hypergroups, primarily because in a hypergroup, the product of two points need not be another point, so the $g_{j} * x_{0}$ may not be contained in a single part of the partition. We define $\xi_{0}(C)$, a measurable function on $H$ which in the group case is just the characteristic function on $x_{0}(C)$. With this approach we are able to give a characterization of amenability for hypergroups, which was inspired by the result of Rosenblatt and Willis for locally compact groups.

Definition 2.1. Let $H$ be a hypergroup with left Haar measure $\lambda$.

Let $\mathcal{E}=\left\{E_{1}, \ldots, E_{m}\right\}$ be a finite measurable partition of $H$ and choose an $n$-tuple of elements of $H, \mathfrak{h}=\left\{h_{1}, \ldots, h_{n}\right\}$. A configuration is an $(n+1)$-tuple $C=\left(C_{0}, C_{1}, \ldots, C_{n}\right)$, where each $C_{j} \in\{1, \ldots, m\}$.

For a fixed configuration, $C$, we define $\xi_{0}(C)$ to be the real-valued function on $H$ given by

$$
\xi_{0}(C)(x):=\prod_{j=0}^{n} \delta_{h_{j}} * \delta_{x}\left(E_{C_{j}}\right)
$$

using the convention that $h_{0}=e$. In particular, if $x \in C_{0}$ and each of $\left\{h_{j}\right\} *\{x\} \subset$ $E_{C_{j}}$, then $\xi_{0}(C)(x)=1$.

An alternate expression for $\xi_{0}(C)$ is

$$
\xi_{0}(C)=\prod_{j=0}^{n} \delta_{\check{h_{j}}} * \chi_{E_{C_{j}}} .
$$


From this we see that $\xi_{0}(C)$ is the pointwise product of finitely many non-negative measurable functions bounded by 1 and so is itself in $L^{\infty}(H)^{+}$and is norm bounded by 1 .

For $f \in L^{1}(H)$ and a configuration $C$, let $f_{C}$ denote the integral

$$
f_{C}:=\int_{H} \xi_{0}(C)(t) f(t) d \lambda(t)
$$

We denote by $\operatorname{Con}(\mathcal{E}, \mathfrak{h})$ the family of configurations associated to that particular choice of $\mathcal{E}$ and $\mathfrak{h}$.

Lemma 2.2. Let $H$ be a hypergroup with left Haar measure $\lambda$. Let $\mathcal{E}$ and $\mathfrak{h}$ be as above. For $f \in L^{1}(H), i \in\{1, \ldots, m\}$ and $j \in\{1, \ldots, n\}$, we have that

$$
\int_{E_{i}} f d \lambda=\sum_{\substack{C \in \operatorname{Con}(\mathcal{E}, \mathfrak{h}) \\ C_{0}=i}} f_{C} \text { and } \int_{E_{i}} \delta_{h_{j}} * f d \lambda=\sum_{\substack{C \in \operatorname{Con}(\mathcal{E}, \mathfrak{h}) \\ C_{j}=i}} f_{C} .
$$

Proof. First, notice that for $x \in H$,

$$
\begin{aligned}
\sum_{\substack{C \in \operatorname{Con}(\mathcal{E}, \mathfrak{h}) \\
C_{0}=i}} \xi_{0}(C)(x) & =\sum_{\substack{C \in \operatorname{Con}(\mathcal{E}, \mathfrak{h}) \\
C_{0}=i}}\left(\prod_{l=0}^{n} \delta_{h_{l}} * \delta_{x}\left(E_{C_{l}}\right)\right) \\
& =\chi_{E_{i}}(x) \prod_{l=1}^{n}\left(\sum_{k=1}^{m} \delta_{h_{l}} * \delta_{x}\left(E_{k}\right)\right) \\
& =\chi_{E_{i}}(x) \prod_{l=1}^{n} \delta_{h_{l}} * \delta_{x}(H) \\
& =\chi_{E_{i}}(x) .
\end{aligned}
$$

So, by integrating $f$ multiplied by the above function, we get

$$
\begin{aligned}
\int_{E_{i}} f d \lambda & =\int_{H} \chi_{E_{i}}(x) f(x) d \lambda(x) \\
& =\int_{H} \sum_{\substack{C \in \operatorname{Con}(\mathcal{E}, \mathfrak{h}) \\
C_{0}=i}} \xi_{0}(C)(x) f(x) d \lambda(x) \\
& =\sum_{\substack{C \in \operatorname{Con}(\mathcal{E}, \mathfrak{h}) \\
C_{0}=i}} \int_{H} \xi_{0}(C)(x) f(x) d \lambda(x) \\
& =\sum_{\left.C \in \operatorname{Con}_{C_{0}=i}, \mathfrak{h}\right)} f_{C} .
\end{aligned}
$$


For the second equality, we again need to rearrange the sum of products to be the product of a sum. Indeed, for $x \in H$,

$$
\begin{aligned}
\sum_{\substack{C \in \operatorname{Con}(\mathcal{E}, \mathfrak{h}) \\
C_{j}=i}} \xi_{0}(C)(x) & =\sum_{\substack{C \in \operatorname{Con}(\mathcal{E}, \mathfrak{h}) \\
C_{j}=i}} \prod_{l=0}^{n} \delta_{h_{l}} * \delta_{x}\left(E_{C_{l}}\right) \\
& =\delta_{h_{j}} * \delta_{x}\left(E_{i}\right) \prod_{\substack{l=0 \\
l \neq j \\
l \neq j}}^{n} \sum_{k=1}^{m} \delta_{h_{l}} * \delta_{x}\left(E_{k}\right) \\
& =\chi_{E_{i}}\left(h_{j} * x\right) .
\end{aligned}
$$

Therefore,

$$
\begin{aligned}
\int_{E_{i}} \delta_{h_{j}} * f d \lambda & =\int_{H} \chi_{E_{i}}\left(h_{j} * t\right) f(t) d \lambda(t) \\
& =\int_{H} \sum_{\substack{C \in \operatorname{Con}(\mathcal{E}, \mathfrak{h}) \\
C_{j}=i}} \xi_{0}(C)(t) f(t) d \lambda(t) \\
& =\sum_{\substack{C \in \operatorname{Con}(\mathcal{E}, \mathfrak{h}) \\
C_{j}=i}} \int_{H} \xi_{0}(C)(t) f(t) d \lambda(t) \\
& =\sum_{\left.C \in \operatorname{Con}_{C_{j}=i}, \mathfrak{h}\right)} f_{C} .
\end{aligned}
$$

Remark 2.1. We see from the above that summing over ALL configurations gives

$$
\sum_{C \in \operatorname{Con}(\mathcal{E}, \mathfrak{h})} \xi_{0}(C)(x)=1 \quad \forall x \in H .
$$

Corollary 2.3. Given $f \in L^{1}(H)$, we have for all $i \in\{1, \ldots, m\}$ and $j \in$ $\{1, \ldots, n\}$,

$$
\left\langle f-\delta_{h_{j}} * f, \chi_{E_{i}}\right\rangle=0
$$

if and only if for all $i \in\{1, \ldots, m\}$ and $j \in\{1, \ldots, n\}$,

$$
\sum_{\substack{C \in \operatorname{Con}(\mathcal{E}, \mathfrak{h}) \\ C_{0}=i}} f_{C}=\sum_{\substack{C \in \operatorname{Con}(\mathcal{E}, \mathfrak{h}) \\ C_{j}=i}} f_{C} .
$$

Rather than start with some $f \in L^{1}(H)$ that generates the values $f_{C}$ which satisfy the equations in the above corollary, we can consider those equations and the solutions to them.

Definition 2.4. Fix $\mathcal{E}$ and $\mathfrak{h}$ as before. Let $\left\{z_{C}: C \in \operatorname{Con}(\mathcal{E}, \mathfrak{h})\right\}$ be variables corresponding to the $m^{n+1}$ configurations. Consider the $m \times n$ configuration equations

$$
\sum_{\substack{C \in \operatorname{Con}(\mathcal{E}, \mathfrak{h}) \\ C_{0}=i}} z_{C}=\sum_{\substack{C \in \operatorname{Con}(\mathcal{E}, \mathfrak{h}) \\ C_{j}=i}} z_{C}
$$

for each $i \in\{1, \ldots, m\}$ and $j \in\{1, \ldots, n\}$. 
We say that a solution to these configuration equations is

- positive if, for each $C \in \operatorname{Con}(\mathcal{E}, \mathfrak{h}), z_{C} \geq 0$;

- normalized if $\sum_{C \in \operatorname{Con}(\mathcal{E}, \mathfrak{h})} z_{C}=1$; and

- inequality preserving if for every choice of $m^{n+1}$ real numbers $\left\{a_{C}: C \in\right.$ $\operatorname{Con}(\mathcal{E}, \mathfrak{h})\}$,

$$
0 \leq \sum_{C \in \operatorname{Con}(\mathcal{E}, \mathfrak{h})} a_{C} \xi_{0}(C) \text { a.e. } \Rightarrow 0 \leq \sum_{C \in \operatorname{Con}(\mathcal{E}, \mathfrak{h})} a_{C} z_{C},
$$

i.e., any inequality which is satisfied by a linear combination of the functions $\xi_{0}(C)$ is also satisfied by the same linear combination of the values of the $z_{C} \mathrm{~s}$.

Clearly, if there exists some $f \in L^{1}(H)_{1}^{+}$for which $\left\langle f-\delta_{h_{j}} * f, \chi_{E_{i}}\right\rangle=0$ for all $i, j$, then $z_{C}=f_{C}$ is a positive, normalized, inequality preserving solution to these configuration equations. We will show in Theorem 2.6 that $H$ is amenable precisely when such solutions to the configuration equations exist for all choices of $m, n, \mathcal{E}$ and $\mathfrak{h}$.

Lemma 2.5. Let $(X, \mu)$ be a measure space. Let $\left(f_{\alpha}\right)_{\alpha \in \Lambda}$ be a finite family of non-negative functions in $L^{\infty}(X, \mu)$ such that $\sum_{\alpha} f_{\alpha}=\chi_{X}$.

Suppose that there are associated $\left(c_{\alpha}\right)_{\alpha \in \Lambda}$ non-negative real numbers such that for any choice of real numbers $\left(a_{\alpha}\right)_{\alpha \in \Lambda}$ if

$$
0 \leq \sum_{\alpha} a_{\alpha} f_{\alpha} \text { almost everywhere, }
$$

the associated inequality

$$
0 \leq \sum_{\alpha} a_{\alpha} c_{\alpha}
$$

also holds.

Then there exists $\hat{\Gamma} \in\left(L^{\infty}(X, \mu)^{*}\right)^{+}$such that $\hat{\Gamma}\left(f_{\alpha}\right)=c_{\alpha}$ for all $\alpha$. Furthermore, $\|\hat{\Gamma}\|=\sum c_{\alpha}$.

Proof. Let $Y=\operatorname{span}\left\{f_{\alpha}: \alpha \in \Lambda\right\}$. Then $Y$ is a finite dimensional (hence closed) subspace of $L^{\infty}(X, \mu)$. Indeed, there is some subset $\Lambda_{0}$ of $\Lambda$ such that $\left\{f_{\alpha}: \alpha \in \Lambda_{0}\right\}$ is a basis for $Y$.

Define $\Gamma: Y \rightarrow \mathbb{R}$ by letting $\Gamma\left(f_{\alpha}\right)=c_{\alpha}$ for $\alpha \in \Lambda_{0}$ and extending it linearly to all of $Y$.

Then for every $\alpha^{\prime} \in \Lambda \backslash \Lambda_{0}$ there exist some real numbers $\left(a_{\alpha}\right)_{\alpha \in \Lambda_{0}}$ such that

$$
f_{\alpha^{\prime}}=\sum_{\alpha \in \Lambda_{0}} a_{\alpha} f_{\alpha}
$$

So, by (2.2), the corresponding equality holds:

$$
c_{\alpha^{\prime}}=\sum_{\alpha \in \Lambda_{0}} a_{\alpha} c_{\alpha},
$$

and we see that $\Gamma\left(f_{\alpha}\right)=c_{\alpha}$ for all $\alpha \in \Lambda$.

Define $\rho: L^{\infty}(X, \mu) \rightarrow \mathbb{R}^{+}$via

$$
\rho(f):=\inf \left\{\sum_{\alpha \in \Lambda} a_{\alpha} c_{\alpha}: a_{\alpha} \in \mathbb{R}^{+}, \sum_{\alpha \in \Lambda} a_{\alpha} f_{\alpha} \geq|f|\right\} .
$$


Claim. $\rho$ is a well-defined seminorm on $L^{\infty}(X, \mu)$.

Since $\sum_{\alpha \in \Lambda} f_{\alpha}=\chi_{X}$, for any $f \in L^{\infty}(X, \mu), \rho(f) \leq \sum_{\alpha \in \Lambda} c_{\alpha}\|f\|_{\infty}$.

Let $f, g \in L^{\infty}(X, \mu), a \in \mathbb{R}$. Then

$$
\begin{aligned}
& \rho(f)+\rho(g)=\inf \left\{\sum_{\alpha \in \Lambda} a_{\alpha} c_{\alpha}: a_{\alpha} \in \mathbb{R}^{+}, \sum_{\alpha \in \Lambda} a_{\alpha} f_{\alpha} \geq|f|\right\} \\
& +\inf \left\{\sum_{\alpha \in \Lambda} b_{\alpha} c_{\alpha}: b_{\alpha} \in \mathbb{R}^{+}, \sum_{\alpha \in \Lambda} b_{\alpha} f_{\alpha} \geq|g|\right\} \\
& \geq \inf \left\{\sum_{\alpha \in \Lambda}\left(a_{\alpha}+b_{\alpha}\right) c_{\alpha}: a_{\alpha}, b_{\alpha} \in \mathbb{R}^{+}, \sum_{\alpha \in \Lambda}\left(a_{\alpha}+b_{\alpha}\right) f_{\alpha} \geq|f|+|g|\right\} \\
& \geq \inf \left\{\sum_{\alpha \in \Lambda}\left(a_{\alpha}\right) c_{\alpha}: a_{\alpha} \in \mathbb{R}^{+}, \sum_{\alpha \in \Lambda} a_{\alpha} f_{\alpha} \geq|f+g|\right\} \\
& =\rho(f+g) .
\end{aligned}
$$

If $a$ is zero, then we clearly have $\rho(0)=0$. Otherwise, if $a$ is non-zero, then

$$
\begin{aligned}
\rho(a f) & =\inf \left\{\sum_{\alpha \in \Lambda} a_{\alpha} c_{\alpha}: a_{\alpha} \in \mathbb{R}^{+}, \sum_{\alpha \in \Lambda} a_{\alpha} f_{\alpha} \geq|a f|\right\} \\
& =|a| \inf \left\{\sum_{\alpha \in \Lambda} \frac{a_{\alpha}}{|a|} c_{\alpha}: a_{\alpha} \in \mathbb{R}^{+}, \sum_{\alpha \in \Lambda} \frac{a_{\alpha}}{|a|} f_{\alpha} \geq|f|\right\} \\
& =|a| \rho(f) .
\end{aligned}
$$

Hence $\rho$ is a seminorm.

Claim. For all $f \in Y, \Gamma(f) \leq \rho(f)$. Suppose $f \in Y$ and there are real numbers $s_{\alpha}$ for which $f=\sum_{\alpha \in \Lambda_{0}} s_{\alpha} f_{\alpha}$. Hence $\Gamma(f)=\sum_{\alpha \in \Lambda_{0}} s_{\alpha} c_{\alpha}$.

Suppose that for some $\left(a_{\alpha}\right)_{\alpha} \in \mathbb{R}^{+}$we have $|f| \leq \sum_{\alpha \in \Lambda} a_{\alpha} f_{\alpha}$. Then

$$
\begin{aligned}
f & \leq \sum_{\alpha \in \Lambda} a_{\alpha} f_{\alpha}, \\
\sum_{\alpha \in \Lambda_{0}} s_{\alpha} f_{\alpha} & \leq \sum_{\alpha \in \Lambda} a_{\alpha} f_{\alpha}, \\
\sum_{\alpha \in \Lambda_{0}} s_{\alpha} c_{\alpha} & \leq \sum_{\alpha \in \Lambda} a_{\alpha} c_{\alpha}, \\
\Gamma(f) & \leq \sum_{\alpha \in \Lambda} a_{\alpha} c_{\alpha},
\end{aligned}
$$

so by taking the infimum, $\Gamma(f) \leq \rho(f)$.

By the Hahn-Banach Theorem, there exists an extension $\hat{\Gamma}$ to all of $L^{\infty}(X, \mu)$ which is bounded by $\rho$.

Claim. $\hat{\Gamma}$ is positive.

Suppose for contradiction that there exist some $f \in L^{\infty}(X, \mu)^{+}$such that $\hat{\Gamma}(f)<$ 0 . 
Let $a_{\alpha} \in \mathbb{R}^{+}$such that $\sum_{\alpha} a_{\alpha} f_{\alpha} \geq f$. Then $\sum_{\alpha} a_{\alpha} f_{\alpha} \geq \sum_{\alpha} a_{\alpha} f_{\alpha}-f \geq 0$. So

$$
\begin{aligned}
\hat{\Gamma}\left(\sum_{\alpha} a_{\alpha} f_{\alpha}-f\right) & =\hat{\Gamma}\left(\sum_{\alpha} a_{\alpha} f_{\alpha}\right)-\hat{\Gamma}(f) \\
& =\sum_{\alpha} a_{\alpha} c_{\alpha}-\hat{\Gamma}(f) \\
& >\sum_{\alpha} a_{\alpha} c_{\alpha} .
\end{aligned}
$$

But

$$
\begin{aligned}
\hat{\Gamma}\left(\sum_{\alpha} a_{\alpha} f_{\alpha}-f\right) & \leq \rho\left(\sum_{\alpha} a_{\alpha} f_{\alpha}-f\right) \\
& \leq \sum_{\alpha} a_{\alpha} c_{\alpha},
\end{aligned}
$$

which is a contradiction, so $\hat{\Gamma}$ is positive.

Since $\sum_{\alpha} f_{\alpha}=\chi_{X}$, it follows that $\hat{\Gamma}$ has norm $\sum_{\alpha} c_{\alpha}$.

We are now ready to give a characterization, using configurations, of amenability of a hypergroup with a left Haar measure.

Remark 2.2. If there is no left Haar measure on $H$, then there is no straightforward way to describe measurable partitions of $H$. However, as we remark in section 5 , we can consider partitions of the identity into continuous functions and use a similar approach to describe amenability using $C(H)$ in place of $L^{\infty}(H)$.

Theorem 2.6. Let $H$ be a hypergroup with left Haar measure $\lambda$. $H$ is amenable if and only if for all choices of $m, n, \mathfrak{h}$ and $\mathcal{E}$ the $m \times n$ configuration equations have a positive, normalized, inequality preserving solution.

Proof. Assume that $H$ is amenable. Then there is a left invariant mean $m$ on $L^{\infty}(H)$. For a configuration $C$, let $z_{C}:=m\left(\xi_{0}(C)\right)$. By equation (2.1) and linearity of $m$, it follows that for any $i, j$,

$$
\sum_{\substack{C \in \operatorname{Con}(\mathcal{E}, \mathfrak{h}) \\ C_{0}=E_{i}}} z_{C}=m\left(\chi_{E_{i}}\right)
$$

and

$$
\sum_{\substack{C \in \operatorname{Con}(\mathcal{E}, \mathfrak{h}) \\ C_{j}=E_{i}}} z_{C}=m\left(\delta_{\check{h_{j}}} * \chi_{E_{i}}\right) .
$$

Since $m$ is left invariant, these are equal, and so the configuration equations are satisfied by this choice of $z_{C}$. It is also apparent that because $m$ is a mean, each $z_{C}$ is non-negative and $\sum_{C} z_{C}=1$. Since $m$ is positive, it preserves inequalities satisfying the last requirement.

For the converse, we consider a choice of $\mathcal{E}$ and $\mathfrak{h}$ and apply Lemma 2.5 to $(H, \lambda),\left\{\xi_{0}(C): C \in \operatorname{Con}(\mathcal{E}, \mathfrak{h})\right\}$, and $\left\{z_{C}: C \in \operatorname{Con}(\mathcal{E}, \mathfrak{h})\right\}$.

We consider an order on the family of choices of $(\mathcal{E}, \mathfrak{h})$ by saying that $(\mathcal{E}, \mathfrak{h}) \preceq$ $(\mathcal{F}, \mathfrak{k})$ if $\mathcal{F}$ is a refinement of $\mathcal{E}$ and $\mathfrak{h} \subseteq \mathfrak{k}$. Under this order, the family becomes a directed set. By indexing with respect to this directed set and taking the means generated by Lemma 2.5. we get a net of means on $L^{\infty}(H)$ which converge in the 
weak* topology to left invariance. Since the set of means is weak* compact, there is an accumulation point of this net which must be a left invariant mean on $L^{\infty}(H)$, hence $H$ is amenable.

Remark 2.3. It is actually enough to use a collection of $\mathcal{E}$ and $\mathfrak{h}$ which is a directed set under the given ordering which contains each set of a basis of the topology of $H$ in one of the partitions and each $h \in H$.

The preceding result gives a new characterization of amenability for hypergroups. Indeed, similar characterizations can be found for the existence of a topological left invariant mean on Lau algebras (Theorem 4.3) or the existence of a left invariant mean on other function algebras on a hypergroup.

In [36], Rosenblatt and Willis proved a version of Theorem 2.6 for locally compact groups using a more constructive approach.

Corollary 2.7 (Rosenblatt and Willis [36]). Let $G$ be a locally compact group. There is a positive, normalized solution of every possible instance of the essential configuration equations if and only if $G$ is amenable.

Proof. Since $G$ is a group, $\delta_{x} * \chi_{E_{i}}=\chi_{x E_{i}}$, so $\xi_{0}(C)=\chi_{x_{0}(C)}$ for some set $x_{0}(C)$. For two configurations $C \neq C^{\prime}$ the sets $x_{0}(C)$ and $x_{0}\left(C^{\prime}\right)$ are disjoint. Rosenblatt and Willis call a configuration $C$ essential if $\lambda_{G}\left(x_{0}(C)\right)>0$. Because of this, the condition that a solution be inequality preserving is equivalent to $z_{C}=0$ for each non-essential configuration.

For an infinite locally compact amenable group $G$, Rosenblatt and Willis 36 . used their constructive proof of Corollary 2.7 to construct nets of positive, norm one functions $\left\{f_{\alpha}\right\}_{\alpha}$ in $L^{1}(G)$ for which $\delta_{x} * f_{\alpha}-f_{\alpha}$ tends weakly to 0 , but $\left\|\delta_{x} * f_{\alpha}-f_{\alpha}\right\|=$ 2 eventually for every $x \in G \backslash\{e\}$. The key to their proof lies in being able to choose a function which, when integrated against a $\xi_{0}(C)$, yields the corresponding $z_{C}$, yet is supported on a small enough set so that the supports of $f$ and $\delta_{x} * f$ are disjoint. Such a result is impossible in general for hypergroups (see Example 2.12) because translation in a hypergroup is not as clean as translation in a group. However, the approach of [36] is helpful for constructing nets for hypergroups which tend to left invariance in a weak sense but not in norm, as demonstrated below in Theorem 2.10 .

Theorem 2.8 (Rosenblatt and Willis [36]). If $G$ is an infinite amenable locally compact group, then there exists a net $\left(f_{\alpha}\right)$ in $P(G)$ converging weakly to invariance such that for every $x \in G \backslash\{e\}$, eventually $\left\|f_{\alpha}-\delta_{x} * f_{\alpha}\right\|=2$.

We can show a similar result (Theorem 2.10) for a large class of hypergroups arising as double coset spaces of locally compact groups. To begin, we need the following lemma.

Lemma 2.9. Let $G$ be a locally compact group and $K$ be a compact subgroup of $G$. If $\left(f_{\alpha}\right)$ is a net converging weakly to left invariance in $L^{1}(G)_{1}^{+}$, then $\left(f_{\alpha}^{\circ}\right)$ is a net in $L^{1}(G / / K)_{1}^{+}$satisfying

$$
\left\langle\dot{\circ}_{\alpha}-\delta_{K x K} * \stackrel{\circ}{f}_{\alpha}, \phi\right\rangle \rightarrow 0
$$

for all $\phi \in U C B_{r}(G / / K)$, where $\stackrel{\circ}{f}(K y K)=\int_{K} \int_{K} f(s y t) d \lambda_{K}(s) d \lambda_{K}(t)$. 
Proof. Let $\phi \in U C B_{r}(G / / K)$. By [41, 2.2] there exist $\gamma \in L^{1}(G / / K)$ and $\psi \in$ $L^{\infty}(G / / K)$ such that $\phi=\gamma * \psi$. Let $\gamma_{\circ} \in L^{1}(G)$ and $\psi_{\circ} \in L^{\infty}(G)$ be given by $\gamma_{\circ}(y)=\gamma(K y K)$ and $\psi_{\circ}(y)=\psi(K y K)$. Define $\phi_{\circ}=\gamma_{\circ} * \psi_{\circ}$ in $U C B_{r}(G)$ so $\phi_{\circ}(y)=\int_{G} \gamma(K y z K) \psi\left(K z^{-1} K\right) d \lambda_{G}(z)$. For $y \in G$ we have

$$
\begin{aligned}
\phi(K y K) & =\int_{G} \gamma(K y K * K z K) \psi\left(K z^{-1} K\right) d \lambda_{G}(z) \\
& =\int_{K} \int_{G} \gamma(K(y t) z K) \psi\left(K z^{-1} K\right) d \lambda_{G}(z) d \lambda_{K}(t) \\
& =\int_{K} \phi_{\circ}(y t) d \lambda_{K}(t) \\
& =\phi_{\circ} * \check{\lambda_{K}}(y) .
\end{aligned}
$$

Similarly, for $x, y \in G$ we have $\phi(K x K * K y K)=\int_{K} \phi_{\circ} * \check{\lambda_{K}}(x s y) d \lambda_{K}(s)$.

Then for $x \in G$ and $f \in L^{1}(G)$, we have the following:

$$
\begin{aligned}
& \left\langle\stackrel{\circ}{f}-\delta_{K x K} * \stackrel{\circ}{f}, \phi\right\rangle=\int_{G / / K}\left(\stackrel{\circ}{f}-\delta_{K x K} * \stackrel{\circ}{f}\right)(K y K) \phi(K y K) d \lambda_{G / / K}(K y K) \\
& =\int_{G}\left(\stackrel{\circ}{f}-\delta_{K x K} * \stackrel{\circ}{f}\right)(K y K) \phi(K y K) d \lambda_{G}(y) \\
& =\int_{G} \stackrel{\circ}{f}(K y K)\left(\phi-\check{\delta}_{K x K} * \phi\right)(K y K) d \lambda_{G}(y) \\
& =\iint_{G} \int_{K} f(s y t) d \lambda_{K}(s) d \lambda_{K}(t)\left(\phi-\check{\delta}_{K x K} * \phi\right)(K y K) d \lambda_{G}(y) \\
& =\iint_{K} \int_{K} f(y)\left(\phi-\check{\delta}_{K x K} * \phi\right)\left(K s^{-1} y t^{-1} K\right) d \lambda_{G}(y) d \lambda_{K}(s) d \lambda_{K}(t) \\
& =\int_{G} f(y)(\phi(K y K)-\phi(K x K * K y K)) d \lambda_{G}(y) \\
& =\int_{G} f(y)\left(\phi_{\circ} * \check{\lambda_{K}}(y)-\int_{K} \phi_{\circ} * \check{\lambda_{K}}(x s y) d \lambda_{K}(s)\right) d \lambda_{G}(y) \\
& =\left\langle f, \phi_{\circ} * \check{\lambda_{K}}-\int_{K} \delta_{s^{-1} x^{-1}} * \phi_{\circ} * \check{\lambda_{K}} d \lambda_{K}(s)\right\rangle \text {. }
\end{aligned}
$$

Now, since $\phi_{\circ}$ is in $U C B_{r}(G)$, so is $\phi_{\circ} * \lambda_{K}$. Hence the map from $K$ to $C(G), s \mapsto$ $\delta_{s^{-1} x^{-1}} * \phi_{\circ} * \bar{\lambda}_{K}$ is continuous, so $\int_{K} \delta_{s^{-1} x^{-1}} * \phi_{\circ} * \lambda_{K} d \lambda_{K}(s)$ is in the norm closure

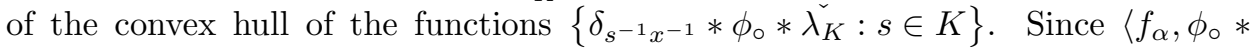
$\left.\check{\lambda_{K}}-\delta_{s^{-1} x^{-1}} * \phi_{\mathrm{o}} * \bar{\lambda}_{K}\right\rangle$ tends to zero for any $s \in K$ and $\left(f_{\alpha}\right)$ is norm bounded, it follows that $\left\langle f_{\alpha}, \phi_{\circ} * \check{\lambda_{K}}-\int_{K} \delta_{s^{-1} x^{-1}} * \phi_{\circ} * \check{\lambda_{K}} d \lambda_{K}(s)\right\rangle$ also tends to zero (see e.g. [35, VI.1.2]). So $\left\langle\dot{f}_{\alpha}-\delta_{K x K} * \stackrel{\circ}{f}_{\alpha}, \phi\right\rangle \rightarrow 0$ for all $\phi \in U C B_{r}(G / / K)$ and all $x \in G$.

Theorem 2.10. Let $G$ be an amenable, non-compact, locally compact group. Let $K$ be a compact subgroup of $G$. Suppose that for any $\varepsilon>0$, finite $F \subset G \backslash K$ and subset $X$ of $G$ which does not have zero measure outside of a compact set, we can 
find a relatively compact $X^{\prime} \subset X$ such that

$$
\lambda_{G}\left(K F K X^{\prime} K \cap K X^{\prime} K\right)<\frac{\varepsilon}{2} \lambda_{G}\left(K X^{\prime} K\right) .
$$

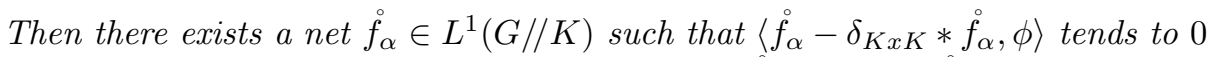
for all $\phi \in U C B_{r}(G / / K)$ and $x \in G$ but for which $\left\|f_{\alpha}^{\circ}-\delta_{K x K} * f_{\alpha}^{\circ}\right\| \rightarrow 2$ whenever $x \notin K$.

Proof. Fix $\varepsilon>0$ and a finite subset $F \subset G \backslash K$ and take $\mathcal{E}$ and $\mathfrak{g}$ as before for the group $G$.

Since $G$ is amenable, there is a positive, normalized solution to the configurations corresponding to $\operatorname{Con}(\mathcal{E}, \mathfrak{g})$.

Since $G$ is a group, for each $C \in \operatorname{Con}(\mathcal{E}, \mathfrak{g})$ the function $\xi_{0}(C)$ is the characteristic function of the set $X_{0}(C)$. If the value of $z_{C}$ is non-zero, then because $G$ is noncompact $X_{0}(C)$ does not have measure zero outside any compact set.

Choose an order $\left\{C_{a}\right\}_{a=1}^{N}$ for the $C \in \operatorname{Con}(\mathcal{E}, \mathfrak{g})$ with non-zero $z_{C}$ and then iteratively select relatively compact $X_{0}^{C} \subset X_{0}(C)$ satisfying inequality (2.3) such that

$$
F K X_{0}^{C_{a}} \cap K X_{0}^{C_{b}} K=\emptyset, a \neq b .
$$

This is possible since each $X_{0}\left(C_{a}\right)$ has non-zero measure outside the compact set

$$
\bigcup_{b=1}^{a-1}\left(K F K X_{0}^{C_{b}} K \cup K F^{-1} K X_{0}^{C_{b}} K\right)
$$

As in [36, 3.2], let

$$
f=\sum_{s=1}^{N} \frac{z_{C_{s}}}{\lambda_{G}\left(X_{0}^{C_{s}}\right)} \chi_{X_{0}^{C_{s}}} .
$$

Then $f \in L^{1}(G)_{1}^{+}$and $\left\langle f-\delta_{g} * f, \chi_{E}\right\rangle=0$ for each $g \in \mathfrak{g}$ and $E \in \mathcal{E}$. Let $\stackrel{\circ}{f} \in L^{1}(G / / K)$ be as in Lemma 2.9 .

Observe that for $y \in G$,

$$
\stackrel{\circ}{f}(K y K)=\sum_{C \in \operatorname{Con}(\mathcal{E}, \mathfrak{g})} \frac{z_{C}}{\lambda_{G}\left(X_{0}^{C}\right)} \int_{K} \int_{K} \chi_{X_{0}^{C}}(s y t) d \lambda_{K}(s) d \lambda_{K}(t),
$$

and if $\stackrel{\circ}{f}(K y K)>0$, then $y \in K X_{0}^{C} K$ for some $C$.

Similarly, for $x \in F, \stackrel{\circ}{f}\left(K x^{-1} K * K y K\right)$ is equal to

$$
\sum_{C \in \operatorname{Con}(\mathcal{E}, \mathfrak{g})} \frac{z_{C}}{\lambda_{G}\left(X_{0}^{C}\right)} \iint_{K} \int_{K} \chi_{X_{0}^{C}}\left(r x^{-1} s y t\right) d \lambda_{K}(r) d \lambda_{K}(s) d \lambda_{K}(t),
$$

and if $\stackrel{\circ}{f}\left(K x^{-1} K * K y K\right)>0$, then $y \in K F K X_{0}^{C} K$ for some $C$.

So, by condition (2.4), if both $\stackrel{\circ}{f}(K y K)>0$ and $\stackrel{\circ}{f}\left(K x^{-1} K * K y K\right)>0$ for some $x \in F$, then there is a unique $C \in \operatorname{Con}(\mathcal{E}, \mathfrak{g})$ with $y \in K X_{0}^{C} K \cap K F K X_{0}^{C} K$.

For each $C \in \operatorname{Con}(\mathcal{E}, \mathfrak{g})$, let

$$
A_{C}=\left\{y \in K X_{0}^{C} K \cap K F K X_{0}^{C} K: \stackrel{\circ}{f}(K y K)>0, \stackrel{\circ}{f}(K x K * K y K)>0\right\} .
$$


This yields

$$
\begin{aligned}
& \int_{A_{C}}\left|f(K y K)-f\left(K x^{-1} K * K y K\right)\right| d \lambda_{G}(y) \\
& \leq \int_{A_{C}} \frac{z_{C}}{\lambda_{G}\left(X_{0}^{C}\right)}\left|\int_{K^{3}} f(s y t)-f\left(r x^{-1} s y t\right) d \lambda_{K}(r) d \lambda_{K}(s) d \lambda_{K}(t)\right| d \lambda_{G}(y) \\
& \leq \frac{z_{C}}{\lambda_{G}\left(X_{0}^{C}\right)} \int_{K^{3}} \int_{A_{C}}\left|\chi_{X_{0}^{C}}(s y t)-\chi_{X_{0}^{C}}(r x s y t)\right| d \lambda_{G}(y) d \lambda_{K}(r) d \lambda_{K}(s) d \lambda_{K}(t) \\
& \leq \frac{z_{C}}{\lambda_{G}\left(X_{0}^{C}\right)} \int_{K^{3}} \int_{A_{C}}(2) d \lambda_{G}(y) d \lambda_{K}(r) d \lambda_{K}(s) d \lambda_{K}(t) \\
& \leq \frac{2 z_{C}}{\lambda_{G}\left(X_{0}^{C}\right)} \lambda_{G}\left(K X_{0}^{C} K \cap K F K X_{0}^{C} K\right) \\
& <z_{C} \varepsilon
\end{aligned}
$$

By inequality (2.3),

$$
\begin{aligned}
& \int_{G}\left|\stackrel{\circ}{f}-\delta_{K x K} * \stackrel{\circ}{f}\right| d \lambda_{G} \geq\|\stackrel{\circ}{f}\|+\left\|\delta_{K x K} * \stackrel{\circ}{f}\right\|-2 \int_{C} \mid \stackrel{\circ}{f}-\delta_{C} \\
& \geq 2-2 \sum_{C} z_{C} \varepsilon \\
& =2-2 \varepsilon \text {. }
\end{aligned}
$$

Since our choices of $\varepsilon, F, \mathcal{E}$, and $\mathfrak{g}$ were arbitrary, we can find an $f$ as above for each such choice. Now consider the order on $\{(\varepsilon, F, \mathcal{E}, \mathfrak{g})\}$ where

$$
(\varepsilon, F, \mathcal{E}, \mathfrak{g}) \preceq\left(\varepsilon^{\prime}, F^{\prime}, \mathcal{E}^{\prime}, \mathfrak{g}^{\prime}\right)
$$

if $\varepsilon \geq \varepsilon^{\prime}, F \subseteq F^{\prime}, \mathcal{E}^{\prime}$ is a refinement of $\mathcal{E}$, and $\mathfrak{g} \subseteq \mathfrak{g}^{\prime}$.

Using this order, the net $\left(f_{(\varepsilon, F, \mathcal{E}, \mathfrak{g})}\right)$ converges weakly to left invariance on $L^{\infty}(G)$, so by Lemma 2.9 ] $\left\langle\stackrel{\circ}{f}(\varepsilon, F, \mathcal{E}, \mathfrak{g})-\delta_{K x K} * \stackrel{\circ}{f}_{(\varepsilon, F, \mathcal{E}, \mathfrak{g})}, \phi\right\rangle \rightarrow 0$ for all $x \in G$ and $\phi \in U C B_{r}(G / / K)$.

On the other hand, $\left\|\stackrel{\circ}{f}(\varepsilon, F, \mathcal{E}, \mathfrak{g})-\delta_{K x K} * \stackrel{\circ}{f}_{(\varepsilon, F, \mathcal{E}, \mathfrak{g})}\right\| \geq 2-2 \varepsilon$ for all $x \in F$. Hence, for any $x \in G \backslash K,\left\|\stackrel{\circ}{f}(\varepsilon, F, \mathcal{E}, \mathfrak{g})-\delta_{K x K} * \stackrel{\circ}{f}_{(\varepsilon, F, \mathcal{E}, \mathfrak{g})}\right\| \rightarrow 2$.

If $K$ is finite, then the net we construct does tend to left invariance weakly.

Example 2.11. Let $H$ be the hypergroup $(\mathbb{Z}[i] \rtimes\langle i\rangle) / /\langle i\rangle$ where the action of $\langle i\rangle$ on $\mathbb{Z}[i]$ is multiplication. The coset of $a+i b \in \mathbb{Z}[i]$ is the four points $\{a+i b,-b+$ $i a,-a-i b, b-i a\}$. For any finite set $F$, if $\|a+i b\|$ is sufficiently large, $X^{\prime}=\{a+i b\}$ will satisfy inequality (2.3) of Theorem 2.10. Hence, using the method of Theorem 2.10, we can construct a net which tends to left invariance weakly, but not in norm for $(\mathbb{Z}[i] \rtimes\langle i\rangle) / /\langle i\rangle$.

Example 2.12. Let $H$ be the hypergroup $\left(\mathbb{R}^{2}\right)^{\mathbb{T}}$ (equivalently $\left(\mathbb{R}^{2} \rtimes \mathbb{T}\right) / / \mathbb{T}$ ) where the action of the torus on $\mathbb{R}^{2}$ is rotation about the origin. More details on this example can be found in [18 or [4, 1.1.18]. The underlying space of $H$ is $\mathbb{R}^{+}$, and 
for any $f \in L^{1}(H)$ and $x \in H$, the support of the translation of $f$ by $x$ is given by

$$
\operatorname{supp}\left(\delta_{x} * f\right)=\{x\} * \operatorname{supp} f=\bigcup_{y \in \operatorname{supp} f}[|x-y|, x+y] .
$$

From this we see that as long as $\operatorname{supp} f$ is not contained in the interval $[0, x / 2)$, the two supports are not disjoint. Since the support of $f_{\alpha}$ must eventually not be contained in such an interval, if $f_{\alpha}$ tends weakly to left invariance, then the supports of $f_{\alpha}$ and $\delta_{x} * f_{\alpha}$ are not eventually disjoint.

However, this hypergroup does satisfy the condition of Theorem 2.10 so we can construct a net which tends weakly to left invariance against right uniformly continuous functions but does not tend to left invariance in norm.

Singh has further comments on this hypergroup in 4.4.6 of [40].

Remark 2.4. This author conjectures that for every infinite hypergroup there is a net of positive, norm one functions tending to left invariance weakly but not in norm. In [36], Rosenblatt and Willis suggest a possible alternative (non-constructive) method to prove the result for groups by considering a net tending to an extreme point in the set of left-invariant means. It is possible that such a method would also work in the case of hypergroups.

\section{SemidireCt PRODUCTS With HyPERGRoup FACTORS}

The examples concluding the previous section are double coset hypergroups. These hypergroups arise from taking semidirect products of locally compact groups and quotienting by the non-normal factor.

In this section we will present the definition of a semidirect product with a hypergroup factor and several related results. We will conclude the section with several results on amenability of semidirect products.

Remark 3.1. The semidirect product of two hypergroups only makes sense if there is a homomorphism from one hypergroup to a subgroup of the automorphisms of the other (in the case of a direct product, this is the trivial group). Because of this, we will consider semidirect products where one factor is a hypergroup and the other factor is a locally compact group acting as automorphisms on the hypergroup. Hypergroup automorphisms and, more generally, homomorphisms are interesting and have been mentioned in [5] and [20].

Remark 3.2. This definition does not appear as part of the published literature, but does appear in a technical report of Rösler [37. where she applies this construction to Bessel-Kingman hypergroups.

Definition 3.1. Let $H$ be a hypergroup. A homeomorphism $\phi: H \rightarrow H$ is a (hypergroup) automorphism if $\phi\left(e_{H}\right)=e_{H}$, and for $x, y \in H$ and $A \subset H$ a Borel subset we have that $\delta_{x} * \delta_{y}(A)=\delta_{\phi(x)} * \delta_{\phi(y)}(\{\phi(a): a \in A\})$. The collection of automorphisms of $H$ (equipped with the topology of pointwise convergence) form a topological group denoted $\operatorname{Aut}(H)$.

Let $G$ be a locally compact group. Suppose that there exists a continuous group action of $G$ on $H$, that is, a continuous group homomorphism $\tau: G \rightarrow \operatorname{Aut}(H)$. We then define the semidirect product of $G$ and $H$ (with respect to $\tau$ ) as the topological 
space $H \times G$ with a convolution defined by

$$
\delta_{\left(h_{1}, g_{1}\right)} * \delta_{\left(h_{2}, g_{2}\right)}=\delta_{h_{1}} * \delta_{\tau_{g_{1}}\left(h_{2}\right)} \otimes \delta_{g_{1} g_{2}},
$$

where we embed the tensor product $M(H) \otimes M(G)$ into $M(H \times G)$.

With this convolution, $H \rtimes_{\tau} G$ becomes a hypergroup. The identity of $H \rtimes_{\tau} G$ is $\left(e_{H}, e_{G}\right)$, and the involution is given by $(h, g)^{r}=\left(\tau_{g^{-1}}(\check{h}), g^{-1}\right)$.

If we further suppose that $H$ has a left Haar measure $\lambda_{H}$, then for each $g \in G$, the measure on $H, \lambda_{H} \circ \tau_{g}$, is a positive multiple of $\lambda_{H}$. Letting $\delta(g)=\frac{\lambda_{H}}{\lambda_{H} \circ \tau_{g}}$ we get the following left Haar measure on $H \rtimes G$ :

$$
d \lambda_{H \rtimes G}(h, g)=\delta(g) d \lambda_{H}(h) d \lambda_{G}(g) .
$$

Proposition 3.2. Let $G, G^{\prime}$ be locally compact groups and $K$ a compact subgroup of $G$. Suppose that $G^{\prime}$ acts on $G$ and that for each $g^{\prime} \in G^{\prime}, g^{\prime}(K)=K$. Then $\left(G \rtimes G^{\prime}\right) / /\left(K \times e_{G^{\prime}}\right)$ is isomorphic to $(G / / K) \rtimes G^{\prime}$.

Proof. The elements of $\left(G \rtimes G^{\prime}\right) / /\left(K \times e_{G^{\prime}}\right)$ are of the form

$$
\begin{aligned}
{\left[\left(g, g^{\prime}\right)\right] } & =\left\{\left(k_{1}, e_{G^{\prime}}\right)\left(g, g^{\prime}\right)\left(k_{2}, e_{G^{\prime}}\right): k_{1}, k_{2} \in K\right\} \\
& =\left\{\left(k_{1} g \tau_{g^{\prime}}\left(k_{2}\right), g^{\prime}\right): k_{1}, k_{2} \in K\right\} \\
& =\left\{\left(k_{1} g k_{2}, g^{\prime}\right): k_{1}, k_{2} \in K\right\}
\end{aligned}
$$

because the action of $G^{\prime}$ restricts to $K$.

Similarly, the elements of $G / / K \rtimes G^{\prime}$ are of the form

$$
\left([g], g^{\prime}\right)=\left(\left\{k_{1} g k_{2}: k_{1}, k_{2} \in K\right\}, g^{\prime}\right),
$$

so there is a natural identification between the two hypergroups.

The multiplication in the former is given by

$$
\begin{aligned}
{\left[\left(g_{1}, g_{1}^{\prime}\right)\right] *\left[\left(g_{2}, g_{2}^{\prime}\right)\right] } & =\int_{K \times e_{G^{\prime}}} \delta_{\left[\left(g_{1}, g_{1}^{\prime}\right)\left(s, e_{G^{\prime}}\right)\left(g_{2}, g_{2}^{\prime}\right)\right]} d \lambda_{K}(s) \\
& =\int_{K \times e_{G^{\prime}}} \delta_{\left[\left(g_{1} \tau_{g_{1}^{\prime}}\left(s g_{2}\right), g_{1}^{\prime} g_{2}^{\prime}\right)\right]} d \lambda_{K}(s) \\
& =\int_{K \times e_{G^{\prime}}} \delta_{\left[\left(g_{1} s \tau_{g_{1}^{\prime}}\left(g_{2}\right), g_{1}^{\prime} g_{2}^{\prime}\right)\right]} d \lambda_{K}(s) .
\end{aligned}
$$

We note that $\lambda_{K}$ is invariant under the action of $g_{1}^{\prime}$ because the action on $K$ is 'unimodular' since $K$ is compact.

On the latter, the convolution is

$$
\begin{aligned}
\left(\left[g_{1}\right], g_{1}^{\prime}\right) *\left(\left[g_{2}\right], g_{2}^{\prime}\right) & =\left(\left[g_{1}\right] * \tau_{g_{1}^{\prime}}\left(\left[g_{2}\right]\right)\right) \otimes g_{1}^{\prime} g_{2}^{\prime} \\
& =\left(\int_{K} \delta_{\left[g_{1} s \tau_{g_{1}^{\prime}}\left(g_{2}\right)\right]} d \lambda_{K}(s)\right) \otimes g_{1}^{\prime} g_{2}^{\prime},
\end{aligned}
$$

and so we see that they coincide.

Remark 3.3. The first semidirect product in the above proposition is a semidirect product of groups, while the latter has a hypergroup factor.

Example 3.3. Here we provide a non-trivial example of a semidirect product with a hypergroup factor. That is, the hypergroup is not a group and the action of $G$ on $H$ is not the trivial action.

Let $\mathbb{Z}_{5}$ be the additive group of integers modulo 5 . Let $\left\{e_{\tau}, \tau\right\}$ be the two element group acting on $\mathbb{Z}_{5}$ via $\tau(z)=-z$. Then $\mathbb{Z}_{5} / /\langle\tau\rangle$ is a hypergroup of three elements. 
Let $\left\{e_{\sigma}, \sigma\right\}$ be the two element group acting on $\mathbb{Z}_{5} / /\langle\tau\rangle$ which swaps the two non-identity elements. It is straightforward to verify that $\sigma$ is a hypergroup automorphism.

Forming the semidirect product $\left(\mathbb{Z}_{5} / /\langle\tau\rangle\right) \rtimes\langle\sigma\rangle$, we get a six element hypergroup.

We now address amenability of semidirect products. As mentioned in Remark 1.4, the amenability of a hypergroup can be characterized in a number of ways. One of these ways is the existence of a Reiter net of approximate means in $L^{1}(H)_{1}^{+}$. It is well known that the semidirect product of two locally compact groups is again amenable, and explicit methods are given in [46] for constructing Reiter nets for a semidirect product. Using that approach, we show that the semidirect product of an amenable hypergroup and an amenable locally compact group is amenable.

Theorem 3.4. Let $H$ be an amenable hypergroup with left Haar measure and $G$ be an amenable locally compact group. Then $H \rtimes_{\tau} G$ is an amenable hypergroup.

Proof. Let $\left(f_{\alpha}\right)$ be a Reiter net for $H$ and $\left(d_{\beta}\right)$ be a Reiter net for $G$ with each $d_{\beta}$ supported on a compact subset of $G$.

For $h \in H, g \in G$ let $F_{\alpha, \beta} \in L^{1}\left(H \rtimes_{\tau} G\right)$ be given by

$$
F_{\alpha, \beta}(h, g):=f_{\alpha}\left(\tau_{g^{-1}}(h)\right) d_{\beta}(g) .
$$

Observe that

$$
\begin{aligned}
& \left\|\delta_{(x, y)} * F_{\alpha, \beta}-F_{\alpha, \beta}\right\|_{L^{1}\left(H \rtimes_{\tau} G\right)} \\
= & \int_{G} \int_{H}\left|f_{\alpha}\left(\tau_{(y g)^{-1}}(\check{x} * h)\right) d_{\beta}\left(y^{-1} g\right)-f_{\alpha}\left(\tau_{g^{-1}}(h)\right) d_{\beta}(g)\right| \delta(g) d \lambda_{H}(h) d \lambda_{G}(g) \\
\leq & \int_{G}\left\|\delta_{\tau_{(y g)^{-1}}(x)} * f_{\alpha}-f_{\alpha}\right\|_{L^{1}(H)}\left|d_{\beta}(g)\right| d \lambda_{G}(g)+\left\|\delta_{y} * d_{\beta}-d_{\beta}\right\|_{L^{1}(G) .}
\end{aligned}
$$

For $K \subset H \rtimes_{\tau} G$ compact and $\varepsilon>0$ there exists $\beta_{K, \varepsilon}$ such that

$$
\left\|\delta_{y} * d_{\beta}-d_{\beta}\right\|_{L^{1}(G)}<\varepsilon / 2 \quad \forall y \text { such that } \exists x \text { with }(x, y) \in K
$$

and there exists $\alpha_{K, \varepsilon}$ such that

$$
\left\|\delta_{\tau(y g)^{-1}(x)} * f_{\alpha_{K, \varepsilon}}-f_{\alpha_{K, \varepsilon}}\right\|<\frac{\varepsilon}{2}
$$

for all $(x, y) \in K$ and $g \in \operatorname{supp}\left(d_{\beta_{K, \varepsilon}}\right)$.

So the semidirect product satisfies the Reiter condition and hence is amenable.

\section{LAU ALGEBRAS}

In 26, Lau introduced a type of Banach algebra (called F algebras in [26]) and defined left amenability of these algebras to correspond to left amenability of the measure algebra of a semigroup. The $L^{1}(H)$ algebra of a hypergroup (with left Haar measure) is a Lau algebra 41 and is left amenable precisely when the hypergroup is amenable. Other examples of Lau algebras include the Fourier and Fourier-Stieljes algebras of locally compact groups. In this section, the constructions of sections 2 and 3 are adapted to the more general setting of Lau algebras. We present a characterization of left amenability using Lau algebra configuration equations. We define the semidirect product of a Lau algebra with a locally compact group and show that this semidirect product is again a Lau algebra. This construction of a 
semidirect product is somewhat similar to the $\theta$-Lau algebra product of 26 and [31. Furthermore, if the Lau algebra factor is left amenable, and the group factor is amenable, then the semidirect product is also left amenable.

Definition 4.1 ([26]). A Lau algebra is a pair $(A, M)$ such that $A$ is a complex Banach algebra and $M$ is a $W^{*}$-algebra such that $A=M_{*}$ and $e$, the identity of $M$, is a multiplicative linear functional on $A$.

Lau, 26] gives several equivalent characterizations of left amenability of $(A, M)$. In particular, the following are equivalent:

(1) The Lau algebra $(A, M)$ is left amenable.

(2) $A^{*}$ has a topological left invariant mean. That is, there exists an $m \in$ $\left(A^{* *}\right)_{1}^{+}$such that

$$
m(x \cdot \phi)=m(x) \quad \forall \phi \in A_{1}^{+}, x \in A^{*} .
$$

(3) There exists a net $\phi_{\alpha} \in A_{1}^{+}$such that $\left\|\phi \cdot \phi_{\alpha}-\phi_{\alpha}\right\| \rightarrow 0$ for each $\phi \in A_{1}^{+}$.

Definition 4.2. Let $(A, M)$ be a Lau algebra. Let $\left(\phi_{1}, \ldots, \phi_{n}\right) \in\left(A_{1}^{+}\right)^{n}$ and $\left\{f_{1}, \ldots, f_{m}\right\} \subset M$ such that each $f_{i} \geq 0$ and $\sum_{i=1}^{m} f_{i}=e_{M}$. We define an $(A, M)$ configuration as an ordered choice $C=\left(C_{0}, C_{1}, \ldots, C_{n}\right)$ with each $c_{j} \in\{1, \ldots, m\}$ and define $\xi_{0}(C)$ as before via

$$
\xi_{0}(C)=\prod_{j=0}^{n} C_{j} \cdot \phi_{j} .
$$

Here the $\cdot$ represents the dual module action of $A$ on $M$. In case the multiplication in $M$ is non-commutative, we need only fix a convention for the ordering and keep to it throughout. For convenience, we'll assume that the multiplication is done left to right as $j$ goes from 0 to $n$.

For $\phi \in A$ we define

$$
\phi_{C}=\left\langle\xi_{0}(C), \phi\right\rangle
$$

We define the configuration equations as before as the $m n$ equations in the $m^{n+1}$ variables $\left(z_{C}\right.$ corresponding to the configuration $C$ ) as

$$
\sum_{C, C_{0}=i} z_{C}=\sum_{C, C_{j}=i} z_{C}
$$

A solution to the configuration equations is again said to be positive if each $z_{C} \geq 0$, normalized if $\sum_{C} z_{C}=1$ and inequality preserving if for any choice of real numbers $\left\{a_{C}\right\}$

$$
0 \leq \sum_{C} a_{C} \xi_{0}(C) \Rightarrow 0 \leq \sum_{C} a_{C} z_{C}
$$

Theorem 4.3. A Lau algebra $(A, M)$ is left amenable if and only if for all choices of $\left(\phi_{1}, \ldots, \phi_{n}\right) \in\left(A_{1}^{+}\right)^{n}$ and $\left\{f_{1}, \ldots, f_{m}\right\} \subset M$ such that each $f_{i} \geq 0$ and $\sum_{i=1}^{m} f_{i}=$ $e_{M}$ the associated $(A, M)$-configuration equations have a positive, normalized, inequality preserving solution. 
Proof. We apply the method of Lemma 2.2 to get

$$
\begin{aligned}
\sum_{C, C_{j}=i} \xi_{0}(C) & =\sum_{C} \prod_{l=0}^{n} f_{C_{l}} \cdot \phi_{l} \\
& =\left(\prod_{l=0}^{j-1} \sum_{k=1}^{m} f_{k} \cdot \phi_{l}\right)\left(f_{i} \cdot \phi_{j}\right)\left(\prod_{l=j}^{n} \sum_{k=1}^{m} f_{k} \cdot \phi_{l}\right) \\
& =\left(\prod_{l} e \cdot \phi_{l}\right) f_{i} \cdot \phi_{j}\left(\prod_{l} e \cdot \phi_{l}\right) \\
& =f_{i} \cdot \phi_{j},
\end{aligned}
$$

so

$$
\left\langle f_{i}, \phi_{j} \cdot \phi\right\rangle=\sum_{C, C_{j}=i} \phi_{C}
$$

for any $i$ and $j$ and $\phi \in A$, noting that any rearrangment is only of a sum and that the order of the multiplication in any term is unchanged.

If $(A, M)$ is left amenable, there exists a topological left invariant mean, $m$, on $M\left[26\right.$. By letting $z_{C}=m\left(\xi_{0}(C)\right)$, we gain a positive, inequality preserving, normalized solution to the configuration equations since

$$
\begin{aligned}
\sum_{C, C_{0}=i} z_{C} & =\sum_{C, C_{0}=i} m\left(\xi_{0}(C)\right) \\
& =m\left(f_{i}\right)=m\left(f_{i} \cdot \phi_{j}\right) \\
& =\sum_{C, C_{j}=f_{i}} m\left(\xi_{0}(C)\right) \\
& =\sum_{C, C_{j}=f_{i}} z_{C} .
\end{aligned}
$$

For the converse, Lemma 2.5 holds with $L^{\infty}(X, \mu)$ replaced by $M$ (with the partial order of $M$ replacing the a.e. ordering of $\left.L^{\infty}(X)\right)$, and we apply the same net construction as we do in Theorem 2.6 to gain a net of means in $\left(A^{* *}\right)_{1}^{+}$which tends weakly to topological left invariance which must have some accumulation point and which is then a topological left invariant mean on $M$.

We now define the notion of a semidirect product of a locally compact group with a Lau algebra. We remark that if the Lau algebra in question is the group algebra of a locally compact group, then the resulting semidirect product corresponds to the group algebra of the semidirect product.

Definition 4.4. Let $G$ be a locally compact group and $(A, M)$ be a Lau algebra. We say that $T$ is an action of $G$ on $(A, M)$ if:

(1) For each $g \in G$ there is an isometric isomorphism $T_{g}: A \rightarrow A$.

(2) The map $g \in G \mapsto T_{g} \in \operatorname{Aut}(A)$ is a continuous group homomorphism.

(3) For each $g \in G$, the dual map $T_{g}^{*}$ is an isometric *-isomorphism of $M$ onto itself.

If $G$ acts on $(A, M)$, then we define the semidirect product of $G$ with $(A, M)$ as the Lau algebra $\left(L_{T}^{1}(G, A), L^{\infty}(G) \bar{\otimes} M\right)$. Here, $L_{T}^{1}(G, A)$ is the Banach space $L^{1}(G, A)$ 
of integrable $A$-valued functions on $G$ with a twisted multiplication. That is, for $F_{1}, F_{2} \in L_{T}^{1}(G, A)$ we define the function $F_{1} * F_{2}$ from $G$ to $A$ by

$$
F_{1} * F_{2}(g)=\int_{h \in G} F_{1}(h) T_{h}\left(F_{2}\left(h^{-1} g\right)\right) d h .
$$

It is well known (e.g. [4]) that $L^{\infty}(G) \bar{\otimes} M$ is indeed a von Neumann algebra and the dual of (the Banach space) $L_{T}^{1}(G, A)$.

Remark 4.1. The multiplication defined above is well defined, and with it, the norm of $L_{T}^{1}(G, A)$ is submultiplicative. To see this, first consider simple tensors $f_{1} \otimes a_{1}, f_{2} \otimes a_{2} \in K(G) \otimes A$. Then for $g \in G$,

$$
f_{1} \otimes a_{1} * f_{2} \otimes a_{2}(g)=\int_{G} f_{1}(h) f_{2}\left(h^{-1} g\right) a_{1} T_{h}\left(a_{2}\right) d h .
$$

So $\operatorname{supp}\left(f_{1} \otimes a_{1} * f_{2} \otimes a_{2}\right)$ is contained in $\operatorname{supp}\left(f_{1}\right) \operatorname{supp}\left(f_{2}\right)$, which is compact. Furthermore, the range of $f_{1} \otimes a_{1} * f_{2} \otimes a_{2} \subset a_{1} T_{\operatorname{supp}\left(f_{1}\right) \operatorname{supp}\left(f_{2}\right)}\left(a_{2}\right)$. Since the map $h \mapsto T_{h}$ is continuous, the range of $f_{1} \otimes a_{1} * f_{2} \otimes a_{2}$ is relatively compact in $A$, so $f_{1} \otimes a_{1} * f_{2} \otimes a_{2} \in L_{T}^{1}(G, A)$.

By linearity, we can extend this argument to all functions in $K(G) \otimes A$.

For $F_{1}, F_{2} \in K(G) \otimes A$,

$$
\begin{aligned}
\left\|F_{1} * F_{2}\right\| & =\int_{g \in G} \int_{h \in G}\left\|F_{1}(h) T_{h}\left(F_{2}\left(h^{-1} g\right)\right)\right\| d h d g \\
& \leq \int_{h \in G}\left\|F_{1}(h)\right\| d h \int_{g \in G}\left\|F_{2}(g)\right\| d g \\
& =\left\|F_{1}\right\|\left\|F_{2}\right\|,
\end{aligned}
$$

and so by density of $K(G) \otimes A$ in $L_{T}^{1}(G, A)$, we conclude that $F_{1} * F_{2} \in L_{T}^{1}(G, A)$ for any $F_{1}, F_{2} \in L_{T}^{1}(G, A)$ and that with this multiplication, $L_{T}^{1}(G, A)$ is a Banach Algebra.

Proposition 4.5. $\left(L_{T}^{1}(G, A), L^{\infty} \bar{\otimes} A^{*}\right)$ is a Lau algebra.

Proof. It is apparent that $L_{T}^{1}(G, A)$ is a Banach algebra and $L^{\infty}(G) \bar{\otimes} A^{*}$ is its dual. Since $L^{\infty}(G)$ and $A^{*}$ are both $W^{*}$ algebras, the tensor product $L^{\infty}(G) \bar{\otimes} A^{*}$ is also a $W^{*}$ algebra with identity $1 \otimes E_{A^{*}}$.

All that remains to show is that $1 \otimes E_{A^{*}}$ is a multiplicative linear functional on $L_{T}^{1}(G, A)$. Let $F_{1}, F_{2} \in L_{T}^{1}(G, A)$. Observe that

$$
\begin{aligned}
\left\langle F_{1} * F_{2}, 1 \otimes e_{A^{*}}\right\rangle & =\int_{G}\left\langle F_{1} * F_{2}(g), e_{A^{*}}\right\rangle d g \\
& =\int_{G} \int_{G}\left\langle F_{1}(h) T_{h} F_{2}\left(h^{-1} g\right), e_{A^{*}}\right\rangle d h d g \\
& =\int_{G} \int_{G}\left\langle F_{1}(h), e_{A^{*}}\right\rangle\left\langle T_{h} F_{2}\left(h^{-1} g\right), e_{A^{*}}\right\rangle d h d g \\
& =\int_{G} \int_{G}\left\langle F_{1}(h), e_{A^{*}}\right\rangle\left\langle F_{2}(g), T_{h}^{*} e_{A^{*}}\right\rangle d g d h \\
& =\int_{G}\left\langle F_{1}(h), e_{A^{*}}\right\rangle d h \int_{G}\left\langle F_{2}(g), e_{A^{*}}\right\rangle d g \\
& =\left\langle F_{1}, 1 \otimes e_{A^{*}}\right\rangle\left\langle F_{2}, 1 \otimes e_{A^{*}}\right\rangle .
\end{aligned}
$$


Lemma 4.6. The positive elements of $L_{T}^{1}(G, A)$ are characterized by

$$
L^{1}(G, A)_{1}^{+}=\left\{F \in L_{T}^{1}(G, A): F(g) \in A_{1}^{+} \text {for almost every } g \in G\right\}
$$

Proof. It is clear that " $\supseteq$ " holds.

To see the converse, suppose that $F \in L_{T}^{1}(G, A)$ such that $\left\{g \in G: F(g) \notin A_{1}^{+}\right\}$ has positive measure. Then we can find $\varepsilon>0$ such that $\left\{g \in G: \exists m \in A^{*},\|m\|=\right.$ $\left.1, \inf _{\alpha \in \mathbb{R}^{+}}\left|\left\langle F(g), m^{*} m\right\rangle-\alpha\right|>\varepsilon\right\}$ has positive measure. Let $K$ be a compact subset of this set with $L:=\lambda(K)>0$. Then we can find a compact $K_{0} \subset K$ such that $F$ is continuous on $K_{0}$ and $\lambda\left(K \backslash K_{0}\right) \leq L / 2$. Pick $x_{0} \in K_{0}$ such that $C:=\left\{g \in K_{0}:\left\|F(g)-F\left(x_{0}\right)\right\|<\varepsilon / 2\right\}$ has positive measure. Let $m_{0} \in A^{*}$ with $\left\|m_{0}\right\|=1$ and $\inf _{\alpha \in \mathbb{R}^{+}}\left|\left\langle F\left(x_{0}\right), m_{0}^{*} m_{0}\right\rangle-\alpha\right|>\varepsilon$. Then we can verify that

$$
\left\langle F,\left(\chi_{C} \otimes m_{0}\right)^{*}\left(\chi_{C} \otimes m_{0}\right)\right\rangle=\int_{C}\left\langle F(g), m_{0}^{*} m_{0}\right\rangle d g
$$

and

$$
\int_{C}\left\langle F\left(x_{0}\right), m_{0}^{*} m_{0}\right\rangle d g=\lambda(C)\left\langle F\left(x_{0}\right), m_{0}^{*} m_{0}\right\rangle .
$$

It follows that

$$
\left|\int_{C}\left\langle F\left(x_{0}\right)-F(g), m_{0}^{*} m_{0}\right\rangle d g\right| \leq \lambda(C) \varepsilon / 2 .
$$

So for $\alpha \in \mathbb{R}^{+}$we have

$$
\begin{aligned}
\mid\left\langle F,\left(\chi_{C}\right.\right. & \left.\left.\otimes m_{0}\right)^{*}\left(\chi_{C} \otimes m_{0}\right)\right\rangle-\alpha \mid \\
& =\left|\int_{C}\left\langle F(g)-F\left(x_{0}\right), m_{0}^{*} m_{0}\right\rangle d g+\int_{C}\left\langle F\left(x_{0}\right), m_{0}^{*} m_{0}\right\rangle d g-\alpha\right| \\
& \geq\left|\lambda(C)\left\langle F\left(x_{0}\right), m_{0}^{*} m_{0}\right\rangle-\alpha\right|-\lambda(C) \varepsilon / 2 .
\end{aligned}
$$

Hence $\left\langle F,\left(\chi_{C} \otimes m_{0}\right)^{*}\left(\chi_{C} \otimes m_{0}\right)\right\rangle$ is not in $\mathbb{R}^{+}$.

Theorem 4.7. Let $G$ be an amenable locally compact group which acts continuously on a left-amenable Lau algebra $(A, M)$. Suppose that $\left(f_{\beta}\right)_{\beta} \subset L^{1}(G)_{1}^{+}$is a Reiter net for $G$ and that $\left(\phi_{\alpha}\right)_{\alpha} \subset A_{1}^{+}$is a net satisfying condition (3) of Definition 4.1. Suppose also that

$$
\left\|T_{g} \phi_{\alpha}-\phi_{\alpha}\right\|_{A} \rightarrow 0
$$

uniformly in $g$ on compact subsets of $G$. For each $\alpha$ and $\beta$, let $F_{\alpha, \beta} \in L_{T}^{1}(G, A)_{1}^{+}$ be given by $F_{\alpha, \beta}(g)=f_{\beta}(g) \phi_{\alpha}$. Then the net $\left(F_{\alpha, \beta}\right)_{\alpha, \beta}$ satisfies condition (3) of Definition 4.1 for $L_{T}^{1}(G, A)$. 
Proof. Fix $\varepsilon>0$. Consider $F \in L^{1}(G, A)_{1}^{+}$.

Then for any $\alpha$ and $\beta$,

$$
\begin{aligned}
& \left\|F * F_{\alpha, \beta}-F_{\alpha, \beta}\right\|_{1}=\int_{G}\left\|F * F_{\alpha, \beta}(g)-F_{\alpha, \beta}(g)\right\| d g \\
& =\int_{G}\left\|\int_{\operatorname{supp}(F)} F(h) T_{h} \phi_{\alpha} f_{\beta}\left(h^{-1} g\right) d h-\phi_{\alpha} f_{\beta}(g)\right\| d g \\
& =\int_{G}\left\|\int_{\operatorname{supp}(F)} F(h) T_{h}\left(\phi_{\alpha}\right) f_{\beta}\left(h^{-1} g\right)-\right\| F(h)\left\|\phi_{\alpha} f_{\beta}(g) d h\right\| d g \\
& \leq \int_{\operatorname{supp}(F)}\|F(h)\|\left(\int_{G}\left\|\frac{F(h)}{\|F(h)\|} T_{h}\left(\phi_{\alpha}\right) f_{\beta}\left(h^{-1} g\right)-f_{\beta}\left(h^{-1} g\right) \phi_{\alpha}\right\|\right. \\
& \left.\quad+\left\|f_{\beta}\left(h^{-1} g\right) \phi_{\alpha}-\phi_{\alpha} f_{\beta}(g)\right\| d g\right) d h \\
& =\int_{\operatorname{supp}(F)}\|F(h)\|\left\|\frac{F(h)}{\|F(h)\|} T_{h}\left(\phi_{\alpha}\right)-\phi_{\alpha}\right\| d h \\
& \quad+\int_{\operatorname{supp}(F)}\|F(h)\|\left\|\delta_{h} * f_{\beta}-f_{\beta}\right\| d h .
\end{aligned}
$$

So if we chose a compact $K \subset G$ such that $\int_{G \backslash K}\|F(h)\| d h<\varepsilon$, then we can find $\beta_{0}$ so that if $\beta \geq \beta_{0}$, then $\left\|\delta_{h} * f_{\beta}-f_{\beta}\right\|<\varepsilon$ for all $h \in K$.

Hence

$$
\begin{aligned}
& \int_{G}\|F(h)\|\left\|\delta_{h} * f_{\beta}-f_{\beta}\right\| d h \leq \int_{K}\|F(h)\|\left\|\delta_{h} * f_{\beta}-f_{\beta}\right\| d h \\
& \quad+\int_{G \backslash K}\|F(h)\|\left\|\delta_{h} * f_{\beta}-f_{\beta}\right\| d h \\
&<\varepsilon+2 \varepsilon .
\end{aligned}
$$

Since the map $h \mapsto T_{h^{-1}}\left(\frac{F(h)}{\|F(h)\|}\right)$ is measurable, we can find a compact $K_{0} \subset K$ with $\lambda\left(K \backslash K_{0}\right)<\varepsilon$ and $T_{h^{-1}}\left(\frac{F(h)}{\|F(h)\|}\right)$ continuous on $K_{0}$. Hence we can find an $\alpha_{0}$ such that for all $h \in K_{0}$ and all $\alpha \geq \alpha_{0}$,

$$
\left\|T_{h^{-1}}\left(\frac{F(h)}{\|F(h)\|}\right) \phi_{\alpha}-\phi_{\alpha}\right\|<\varepsilon .
$$

Also, by the assumption, we can find $\alpha_{1} \geq \alpha_{0}$ such that for $\alpha \geq \alpha_{1}$ it follows that $\left\|T_{h} \phi_{\alpha}-\phi_{\alpha}\right\|<\varepsilon$ for all $h \in K_{0}$. 
So for $\alpha \geq \alpha_{1}$,

$$
\begin{aligned}
& \int_{\operatorname{supp}(F)}\|F(h)\|\left\|\frac{F(h)}{\|F(h)\|} T_{h}\left(\phi_{\alpha}\right)-\phi_{\alpha}\right\| d h \\
& \quad \leq \int_{\operatorname{supp}(F)}\|F(h)\|\left(\left\|\frac{F(h)}{\|F(h)\|} T_{h}\left(\phi_{\alpha}\right)-T_{h} \phi_{\alpha}\right\|+\left\|T_{h} \phi_{\alpha}-\phi_{\alpha}\right\|\right) d h \\
& =\int_{K_{0}}\|F(h)\|\left(\left\|T_{h}\right\|\left\|T_{h^{-1}}\left(\frac{F(h)}{\|F(h)\|}\right) \phi_{\alpha}-\phi_{\alpha}\right\|+\left\|T_{h} \phi_{\alpha}-\phi_{\alpha}\right\|\right) d h \\
& \quad+\int_{G \backslash K_{0}}\|F(h)\|\left(\left\|\frac{F(h)}{\|F(h)\|} T_{h}\left(\phi_{\alpha}\right)-T_{h} \phi_{\alpha}\right\|+\left\|T_{h} \phi_{\alpha}-\phi_{\alpha}\right\|\right) d h \\
& <\varepsilon+\varepsilon+4 \varepsilon .
\end{aligned}
$$

Then, for $\alpha \geq \alpha_{1}$ and $\beta \geq \beta_{0}$ we have $\left\|F * F_{\alpha, \beta}-F_{\alpha, \beta}\right\|_{1}<9 \varepsilon$.

Theorem 4.8. Let $G$ be an amenable locally compact group which acts continuously on a left-amenable Lau algebra $(A, M)$. Suppose that $\left(f_{\beta}\right)_{\beta}$ is a Reiter net for $G$ such that $\operatorname{supp}\left(f_{\beta}\right)$ is compact for each $\beta$ and that $\left(\phi_{\alpha}\right)_{\alpha}$ is a net in $A_{1}^{+}$satisfying condition (3) of Definition 4.1. For each $\alpha$ and $\beta$, let $F_{\alpha, \beta} \in L_{T}^{1}(G, A)_{1}^{+}$be given by $F_{\alpha, \beta}(g)=f_{\beta}(g) T_{g}\left(\phi_{\alpha}\right)$. Then there exists a subnet of $\left(F_{\alpha, \beta}\right)_{\alpha, \beta}$ which satisfies condition (3) of Definition 4.1 for $L_{T}^{1}(G, A)$.

Proof. This is analogous to a result from [46]. Fix $\varepsilon>0$ and $F \in L_{T}^{1}(G, A)_{1}^{+}$. Then for any $\alpha$ and $\beta$,

$$
\begin{array}{rl}
\| F & * F_{\alpha, \beta}-F_{\alpha, \beta} \|_{1} \\
& =\int_{G}\left\|\int_{G} F(h) T_{h}\left(T_{h^{-1} g} \phi_{\alpha} f_{\beta}\left(h^{-1} g\right)\right) d h-T_{g} \phi_{\alpha} f_{\beta}(g)\right\| d g \\
\leq & \left\|T_{g}\right\| \int_{G} \int_{G}\left\|T_{g^{-1}}(F(h)) \phi_{\alpha} f_{\beta}\left(h^{-1} g\right)-\right\| F(h)\left\|\phi_{\alpha} f_{\beta}(g)\right\| d h d g \\
\leq & \int_{G} \int_{G}\left\|T_{g^{-1}}(F(h)) \phi_{\alpha} f_{\beta}\left(h^{-1} g\right)-\right\| F(h)\left\|\phi_{\alpha} f_{\beta}\left(h^{-1} g\right)\right\| \\
& \quad+\|F(h)\| \phi_{\alpha} f_{\beta}\left(h^{-1} g\right)-\|F(h)\| \phi_{\alpha} f_{\beta}(g) \| d h d g \\
\leq & \int_{\operatorname{supp}(F)}\|F(h)\| \int_{\operatorname{supp}\left(f_{\beta}\right)}\left\|T_{(h g)^{-1}}\left(\frac{F(h)}{\|F(h)\|}\right) \phi_{\alpha}-\phi_{\alpha}\right\| f_{\beta}(g) d g d h \\
\quad & \quad \int_{\operatorname{supp}(F)}\|F(h)\|\left\|l_{h}^{*} f_{\beta}-f_{\beta}\right\| d h .
\end{array}
$$

Since the compactly supported functions are dense in $L_{T}^{1}(G, A)$, we may assume that $F$ is compactly supported. Since $\left(f_{\beta}\right)_{\beta}$ is a Reiter net, there is a $\beta_{F, \varepsilon}$ such that for $h \in \operatorname{supp}(F),\left\|l_{h}^{*} f_{\beta_{F, \varepsilon}}-f_{\beta_{F, \varepsilon}}\right\|<\varepsilon$.

We can also find an $\alpha_{F, \varepsilon, \beta_{F, \varepsilon}}$ such that for all $g \in \operatorname{supp}\left(f_{\beta_{F, \varepsilon}}\right)$ and $h \in \operatorname{supp}(F)$, $\left\|T_{(h g)^{-1}}\left(\frac{F(h)}{\|F(h)\|}\right) \phi_{\alpha}-\phi_{\alpha}\right\|<\varepsilon$.

Consider the directed set $\Lambda$, where each element of $\Lambda$ is a quadruple consisting of a compactly supported $F \in L_{T}^{1}(G, A)_{1}^{+}$, an $\varepsilon>0$, a $\beta \geq \beta_{F, \varepsilon}$, and an $\alpha \geq \alpha_{F, \varepsilon, \beta}$. 
The order we put on $\Lambda$ is $\preceq$, where $\left(F_{1}, \varepsilon_{1}, \beta_{1}, \alpha_{1}\right) \preceq\left(F_{2}, \varepsilon_{2}, \beta_{2}, \alpha_{2}\right)$ if $\operatorname{supp}\left(F_{1}\right) \subset$ $\operatorname{supp}\left(F_{2}\right), \varepsilon_{1} \geq \varepsilon_{2}, \beta_{1} \leq \beta_{2}$, and $\alpha_{1} \leq \alpha_{2}$. From the above observations, it is apparent that for any $\varepsilon$ and $F$, there exists $\alpha$ and $\beta$ such that $\left\|F * F_{\alpha, \beta}-F_{\alpha, \beta}\right\|_{1}<\varepsilon$.

Then $\left(F_{\alpha, \beta}\right)_{F, \varepsilon, \beta, \alpha}$ is a subnet of $\left(F_{\alpha, \beta}\right)_{\alpha, \beta}$, which is an appropriate net.

Corollary 4.9. The semidirect product of an amenable locally compact group with a left-amenable Lau algebra is again a left-amenable Lau algebra.

\section{Further COMMENTS}

Remark 5.1. The approach of configuration equations can be extended from considering partitions of $H$ into subsets to considering partitions of $\chi_{H}$ into continuous functions and dealing with the existence of a left invariant mean on a space of continuous functions rather than $L^{\infty}(H)$. One motivation for this approach is to characterize amenability without assuming the existence of a left Haar measure.

Let $H$ be a hypergroup and $\mathcal{A}$ be a norm closed subalgebra of $C(H)$ which is closed under left translation, pointwise multiplication, lattice operations (min and $\max )$ and contains the identity. Examples of such algebras include the continuous and bounded functions on $H$, the uniformly continuous functions on $H$ and if $H$ is a locally compact group, the almost periodic functions.

Many of the earlier results apply to such algebras with $\mathcal{A}$ in place of $L^{\infty}(X, \mu)$.

Definition 5.1. Let $\left(h_{1}, \ldots, h_{n}\right) \in H^{n}$ and $\left\{f_{1}, \ldots, f_{m}\right\} \subset \mathcal{A}$ such that each $f_{i} \geq 0$ and $\sum_{i=1}^{n} f_{i}=\chi_{H}$. We define an $\mathcal{A}$-configuration as an ordered choice of the $f_{i} \mathrm{~s}$, $C=\left(c_{0}, c_{1}, \ldots, c_{n}\right)$ and define $\xi_{0}(C)$ as before via

$$
\xi_{0}(C)=\prod_{j=0}^{n} \delta_{\breve{h}_{j}} * c_{j} .
$$

Remark 5.2. There exists a left invariant mean on $\mathcal{A}$ iff for all choices of $\left(h_{1}, \ldots, h_{n}\right)$ $\in H^{n}$ and partitions of $\chi_{H},\left\{f_{1}, \ldots, f_{m}\right\} \subset \mathcal{A}$, the associated $\mathcal{A}$-configuration equations have a positive, normalized, inequality preserving solution.

Locally compact quantum groups and, more generally, Hopf-von Neumann algebras have recently been investigated as interesting generalizations of locally compact groups (e.g. [16, 17]). Indeed, recently Daws and Runde [6] have generalized Reiter's property characterization of amenability to the locally compact quantum group case. Unfortunately, the theory of locally compact quantum groups does not apply to hypergroups.

Remark 5.3. Let $H$ be a hypergroup with left Haar measure $\lambda$. Then $L^{\infty}(H)$ is a Hopf-von Neumann algebra with the co-multiplication induced by convolution on $L^{1}(H)$ if and only if $H$ is a locally compact group.

\section{ACKNOWLEDGEMENTS}

This paper consists of results from the author's doctoral studies under the supervision of Professor Anthony To-Ming Lau at the University of Alberta. The author wishes to thank Professor Lau for providing motivation and many helpful suggestions. Thanks also are due to Kenneth Ross, Ajit Iqbal Singh, and the referee for their helpful comments. 


\section{REFERENCES}

[1] A. Abdollahi, A. Rejali, and G. A. Willis, Group properties characterised by configurations, Illinois J. Math. 48 (2004), no. 3, 861-873. MR2114255(2005k:43001)

[2] A. Azimifard, Hypergroups with unique $\alpha$-means (English, with English and French summaries), C. R. Math. Acad. Sci. Soc. R. Can. 30 (2008), no. 2, 33-39. MR2460049 (2010a:43011)

[3] A. Azimifard, On the $\alpha$-amenability of hypergroups, Monatsh. Math. 155 (2008), no. 1, 1-13, DOI 10.1007/s00605-008-0537-1. MR2434922(2010h:43005)

[4] W. R. Bloom and H. Heyer, Harmonic analysis of probability measures on hypergroups, de Gruyter Studies in Mathematics, vol. 20, Walter de Gruyter \& Co., Berlin, 1995. MR.1312826 (96a:43001)

[5] W. R. Bloom and M. E. Walter, Isomorphisms of hypergroups, J. Austral. Math. Soc. Ser. A 52 (1992), no. 3, 383-400. MR.1151294 (93a:43004)

[6] M. Daws and V. Runde, Reiter's properties $\left(P_{1}\right)$ and $\left(P_{2}\right)$ for locally compact quantum groups, J. Math. Anal. Appl. 364 (2010), no. 2, 352-365, DOI 10.1016/j.jmaa.2009.11.036. MR:2576188(2010m:46110)

[7] C. F. Dunkl, The measure algebra of a locally compact hypergroup, Trans. Amer. Math. Soc. 179 (1973), 331-348. MR0320635 (47 \#9171)

[8] C. F. Dunkl and D. E. Ramirez, A family of countably compact $P_{*}$-hypergroups, Trans. Amer. Math. Soc. 202 (1975), 339-356. MR0380267 (52 \#1167)

[9] F. Ghahramani and R. J. Loy, Generalized notions of amenability, J. Funct. Anal. 208 (2004), no. 1, 229-260, DOI 10.1016/S0022-1236(03)00214-3. MR2034298 (2005c:46065)

[10] F. Ghahramani and A. R. Medgalchi, Compact multipliers on weighted hypergroup algebras, Math. Proc. Cambridge Philos. Soc. 98 (1985), no. 3, 493-500, DOI 10.1017/S0305004100063696. MR803608(87i:43004)

[11] F. Ghahramani and A. R. Medgalchi, Compact multipliers on weighted hypergroup algebras. II, Math. Proc. Cambridge Philos. Soc. 100 (1986), no. 1, 145-149, DOI 10.1017/S0305004100065944. MR838661 (87i:43005)

[12] F. Ghahramani and Y. Zhang, Pseudo-amenable and pseudo-contractible Banach algebras, Math. Proc. Cambridge Philos. Soc. 142 (2007), no. 1, 111-123, DOI 10.1017/S0305004106009649. MR2296395 (2008b:46070)

[13] K. Hartmann, R. W. Henrichs, and R. Lasser, Duals of orbit spaces in groups with relatively compact inner automorphism groups are hypergroups, Monatsh. Math. 88 (1979), no. 3, 229238, DOI 10.1007/BF01295237. MR553732 (81b:43006)

[14] N. Hindman and D. Strauss, Density and invariant means in left amenable semigroups, Topology Appl. 156 (2009), no. 16, 2614-2628, DOI 10.1016/j.topol.2009.04.016. MR2561213 (2011b:43003)

[15] Z. Hu, M. Sangani Monfared, and T. Traynor, On character amenable Banach algebras, Studia Math. 193 (2009), no. 1, 53-78, DOI 10.4064/sm193-1-3. MR2506414 (2010d:46060)

[16] Z. Hu, M. Neufang, and Z.-J. Ruan, On topological centre problems and SIN quantum groups, J. Funct. Anal. 257 (2009), no. 2, 610-640, DOI 10.1016/j.jfa.2009.02.004. MR.2527031 (2011a:46107)

[17] Z. Hu, M. Neufang, and Z.-J. Ruan, Multipliers on a new class of Banach algebras, locally compact quantum groups, and topological centres, Proc. Lond. Math. Soc. (3) 100 (2010), no. 2, 429-458, DOI 10.1112/plms/pdp026. MR2595745(2011c:46104)

[18] R. I. Jewett, Spaces with an abstract convolution of measures, Advances in Math. 18 (1975), no. 1, 1-101. MR0394034 (52 \#14840)

[19] E. Kaniuth, A. T. Lau, and J. Pym, On $\phi$-amenability of Banach algebras, Math. Proc. Cambridge Philos. Soc. 144 (2008), no. 1, 85-96, DOI 10.1017/S0305004107000874. MR2388235 (2009a:46084)

[20] E. Kaniuth, A. T. Lau, and A. Ülger, Homomorphisms of commutative Banach algebras and extensions to multiplier algebras with applications to Fourier algebras, Studia Math. 183 (2007), no. 1, 35-62, DOI 10.4064/sm183-1-3. MR2360256 (2008k:46147)

[21] R. Lasser, Almost periodic functions on hypergroups, Math. Ann. 252 (1980), no. 3, 183-196, DOI 10.1007/BF01420082. MR593632(82d:43007)

[22] R. Lasser, Amenability and weak amenability of $l^{1}$-algebras of polynomial hypergroups, Studia Math. 182 (2007), no. 2, 183-196, DOI 10.4064/sm182-2-6. MR2338484 (2008k:43012) 
[23] R. Lasser, On $\alpha$-amenability of commutative hypergroups, Infinite dimensional harmonic analysis IV, World Sci. Publ., Hackensack, NJ, 2009, pp. 184-195, DOI 10.1142/9789812832825_0013. MR2581596 (2011d:43004)

[24] R. Lasser, Various amenability properties of the $L^{1}$-algebra of polynomial hypergroups and applications, J. Comput. Appl. Math. 233 (2009), no. 3, 786-792, DOI 10.1016/j.cam.2009.02.046. MR.2583017 (2011j:43005)

[25] R. Lasser and M. Skantharajah, Reiter's condition for amenable hypergroups, Monatsh. Math. 163 (2011), no. 3, 327-338, DOI 10.1007/s00605-010-0206-z. MR2805877 (2012e:43007)

[26] A. T.-M. Lau, Analysis on a class of Banach algebras with applications to harmonic analysis on locally compact groups and semigroups, Fund. Math. 118 (1983), no. 3, 161-175. MR736276 (85k:43007)

[27] A. T.-M. Lau, H. Miyake, and W. Takahashi, Approximation of fixed points for amenable semigroups of nonexpansive mappings in Banach spaces, Nonlinear Anal. 67 (2007), no. 4, 1211-1225, DOI 10.1016/j.na.2006.07.008. MR2325374(2008e:47122)

[28] A. T.-M. Lau, N. Shioji, and W. Takahashi, Existence of nonexpansive retractions for amenable semigroups of nonexpansive mappings and nonlinear ergodic theorems in Banach spaces, J. Funct. Anal. 161 (1999), no. 1, 62-75, DOI 10.1006/jfan.1998.3352. MR.1670206 $(99 \mathrm{j}: 47079)$

[29] A. T.-M. Lau and J. C. S. Wong, Invariant subspaces for algebras of linear operators and amenable locally compact groups, Proc. Amer. Math. Soc. 102 (1988), no. 3, 581-586, DOI 10.2307/2047227. MR.928984 (89c:43007)

[30] A. T.-M. Lau and Y. Zhang, Fixed point properties of semigroups of non-expansive mappings, J. Funct. Anal. 254 (2008), no. 10, 2534-2554, DOI 10.1016/j.jfa.2008.02.006. MR2406686 (2010g:47105)

[31] M. S. Monfared, On certain products of Banach algebras with applications to harmonic analysis, Studia Math. 178 (2007), no. 3, 277-294, DOI 10.4064/sm178-3-4. MR2289357 $(2007 \mathrm{~m}: 46068)$

[32] M. S. Monfared, Character amenability of Banach algebras, Math. Proc. Cambridge Philos. Soc. 144 (2008), no. 3, 697-706, DOI 10.1017/S0305004108001126. MR2418712 (2009b:46104)

[33] V. Muruganandam, Fourier algebra of a hypergroup. I, J. Aust. Math. Soc. 82 (2007), no. 1, 59-83, DOI 10.1017/S144678870001747X. MR2301971 (2008c:43004)

[34] I. Namioka, Følner's conditions for amenable semi-groups, Math. Scand. 15 (1964), 18-28. MR0180832 (31 \#5062)

[35] A. P. Robertson and W. Robertson, Topological vector spaces, 2nd ed., Cambridge University Press, London-New York, 1973. Cambridge Tracts in Mathematics and Mathematical Physics, No. 53. MR0350361 (50 \#2854)

[36] J. M. Rosenblatt and G. A. Willis, Weak convergence is not strong convergence for amenable groups, Canad. Math. Bull. 44 (2001), no. 2, 231-241, DOI 10.4153/CMB-2001-023-x. MR:1827857 (2002d:43001)

[37] M. Rösler, Radial wavelets and Bessel-Kingman hypergroups, Tech. report, 1997.

[38] K. A. Ross, Hypergroups and centers of measure algebras, Symposia Mathematica, Vol. XXII (Convegno sull'Analisi Armonica e Spazi di Funzioni su Gruppi Localmente Compatti, INDAM, Rome, 1976), Academic Press, London, 1977, pp. 189-203. MR0511036 (58 \#23344)

[39] K. A. Ross, Centers of hypergroups, Trans. Amer. Math. Soc. 243 (1978), 251-269. MR0493161 (58 \#12192)

[40] A. I. Singh, Completely positive hypergroup actions, Mem. Amer. Math. Soc. 124 (1996), no. 593, xii+68, DOI 10.1090/memo/0593. MR.1355035(97d:43001)

[41] M. Skantharajah, Amenable hypergroups, Illinois J. Math. 36 (1992), no. 1, 15-46. MR:1133768 (92k:43002)

[42] R. Spector, Mesures invariantes sur les hypergroupes (French, with English summary), Trans. Amer. Math. Soc. 239 (1978), 147-165. MR0463806 (57 \#3745)

[43] M. Takesaki, Theory of operator algebras. I, Springer-Verlag, New York-Heidelberg, 1979. MR548728 (81e:46038)

[44] M. Voit, Projective and inductive limits of hypergroups, Proc. London Math. Soc. (3) 67 (1993), no. 3, 617-648, DOI 10.1112/plms/s3-67.3.617. MR.1238047 (94m:43012)

[45] R. C. Vrem, Harmonic analysis on compact hypergroups, Pacific J. Math. 85 (1979), no. 1, 239-251. MR571638 (81g:43009) 
[46] B. Willson, Reiter nets for semidirect products of amenable groups and semigroups, Proc. Amer. Math. Soc. 137 (2009), no. 11, 3823-3832, DOI 10.1090/S0002-9939-09-09957-2. MR.2529892 (2010j:43001)

[47] S. Wolfenstetter, Weakly almost periodic functions on hypergroups, Monatsh. Math. 96 (1983), no. 1, 67-79, DOI 10.1007/BF01298935. MR721597 (85h:43008)

Department of Mathematical and Statistical Sciences, University of Alberta, Edmonton, Alberta, Canada T6G 2G1

Current address: Department of Mathematics, Hanyang University, Seoul, South Korea

E-mail address: bwillson@ualberta.ca 